\title{
OFFICE MEMORANDUM
}

FROM

SUBJECT : Erratum

SYMBOL : ISD-6

Figure 5 on page 3 was printed upside down. 


\title{
ISOTOPE FUELS IMPACT TESTER (IFIT)
}

by

C. E. Frantz, J. W. Taylor, and D. B. Court

\begin{abstract}
The Isotope Fuels Impact Tester (IFIT) is used for safely impacting ${ }^{238} \mathrm{PuO}_{2}$ heat sources that have been heated to $2000^{\circ} \mathrm{C}$. Impact velocities reach $300 \mathrm{~m} / \mathrm{s}(1000 \mathrm{ft} / \mathrm{s})$. A gas gun with a $178-\mathrm{mm}(7-\mathrm{in}$.) bore is used to accelerate the heat source, which is heated by a furnace built into the projectile. Double containment of the impacted heat source is obtained by two vessels that are sealed directly to the gun muzzle. The impact occurs in the inner vessel, and parts of the projectile jam into and thereby close each vessel. The inner vessel, containing the impacted heat source, is removed from the gun and is placed inside a glovebox for disassembly and heat-source recovery.

IFIT's modular structure makes it versatile and adaptable to many types of tests. Many applications have demonstrated its versatility and, more important, its capability for impacting ${ }^{238} \mathrm{PuO}$ heat sources safely.

An approximate theoretical relation is used to predict proper conditions for achieving desired impact velocities. Bore lubricants and projectile-seal design are also important for achieving proper impact velocities.
\end{abstract}

\section{INTRODUCTION}

The Isotope Fuels Impact Tester (IFIT) was developed to perform impact studies on heat sources used in Space Nuclear Auxiliary Power Radioisotope Thermoelectric Generators (SNAP RTSs). ${ }^{1}$ Usually the radioactive isotope is ${ }^{238} \mathrm{Pu}$ because of its convenient half-life of $\sim 89 \mathrm{yr}$ and its moderately large critical mass.* Before radioisotope heat sources ${ }^{*}$ Critical masses of ${ }^{238} \mathrm{Pu}$ are about the same as for fast neutron critical assemblies of ${ }^{239} \mathrm{Pu}$. Critical masses of ${ }^{238} \mathrm{Pu}$ cannot be obtained for thermal neutron assemblies. Consequently, several kilograms of ${ }^{238} \mathrm{Pu}$ can be safely assembled together.

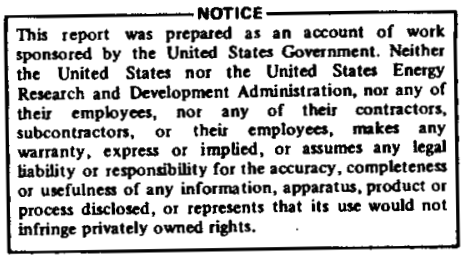

This report was prepared as an account of work sponsored by the United States Goved States Energy Research and Derelopment $\mathrm{Adminlitation,} \mathrm{nor} \mathrm{any} \mathrm{or}$ subcontractors, or their employees, makes any warranty, express or implied, or assumes any legal infringe privately owned rights. 


\section{DISCLAIMER}

This report was prepared as an account of work sponsored by an agency of the United States Government. Neither the United States Government nor any agency Thereof, nor any of their employees, makes any warranty, express or implied, or assumes any legal liability or responsibility for the accuracy, completeness, or usefulness of any information, apparatus, product, or process disclosed, or represents that its use would not infringe privately owned rights. Reference herein to any specific commercial product, process, or service by trade name, trademark, manufacturer, or otherwise does not necessarily constitute or imply its endorsement, recommendation, or favoring by the United States Government or any agency thereof. The views and opinions of authors expressed herein do not necessarily state or reflect those of the United States Government or any agency thereof. 


\section{DISCLAIMER}

Portions of this document may be illegible in electronic image products. Images are produced from the best available original document. 
can be approved for use in space, they must be rigorously tested to insure that adequate fuel containment is achieved by the heat source design. This is done by subjecting the heat source or its component parts to more severe thermal and mechanical environments than the heat source might experience before, during, and after a space flight. Because many of the postulated accident paths include earth impact, impact survivability tests are necessary. The IFIT provides a more reliable test mode because it allows real fuel units to be tested.*

This report describes the IFIT, gives examples of how it has been used, provides the derivation of an approximate theoretical equation for relating muzzle velocity, projectile mass, and breech pressure, and describes practical ways to obtain reproducible projectile velocities.

\section{DESCRIPTION}

IFIT is a gas-driven device used for impacting heated samples that contain high levels of alpha radioactivity. The initial design criteria were:

- Samples containing ${ }^{238} \mathrm{Pu}$ must be impacted safely, even if the primary containment of the ${ }^{238} \mathrm{Pu}$ ruptures on impact.

- Provide means to heat the impact sample up to $2000^{\circ} \mathrm{C}$ just before impact.

- No fasteners should be used to hold the impact sample during a test.

- The device must accommodate many different types of samples and be versatile in its applications.

- Capability to impact samples at velocities up to $\sim 300 \mathrm{~m} / \mathrm{s}(1000 \mathrm{ft} / \mathrm{s})$ is desired.

- Accurate alignment of components for obtaining fundamental material properties is desirable. (Not discussed further in this report.)

The original design provided a device which met the above criteria, and IFIT has been in operation since 1971. The adaptability of this design has enabled us to improve upon the original capabilities by adding modular elements for new test needs.

To achieve versatility, the IFIT was designed as a modular assembly consisting of five major parts: a gas gun, a projectile, a trigger, an impact chamber, and residual-energy-absorbing equipment. Figures $1-3$ are schematic drawings of the breech and muzzle ends of the gun; Figs. 4-6 are pictures of the same components.

*Before IFIT, dummy heat sources loaded with a fuel simulant were tested, and the results were extrapolated to predict the behavior of a system with real fuel. However, the extrapolation was uncertain because of unknown and poorly controlled impact properties of the dummy and fuel systems.

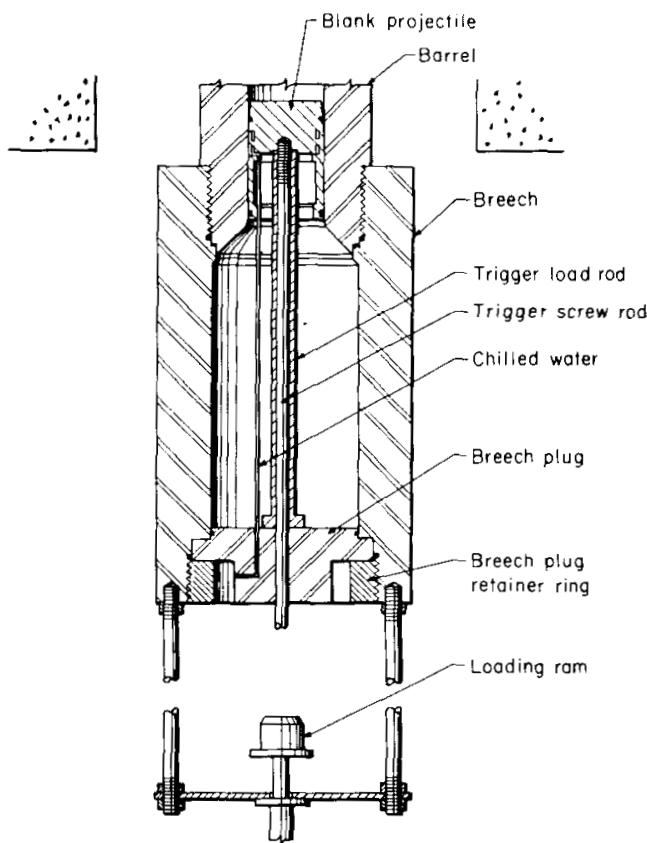

Fig. 1.

Gun breech.

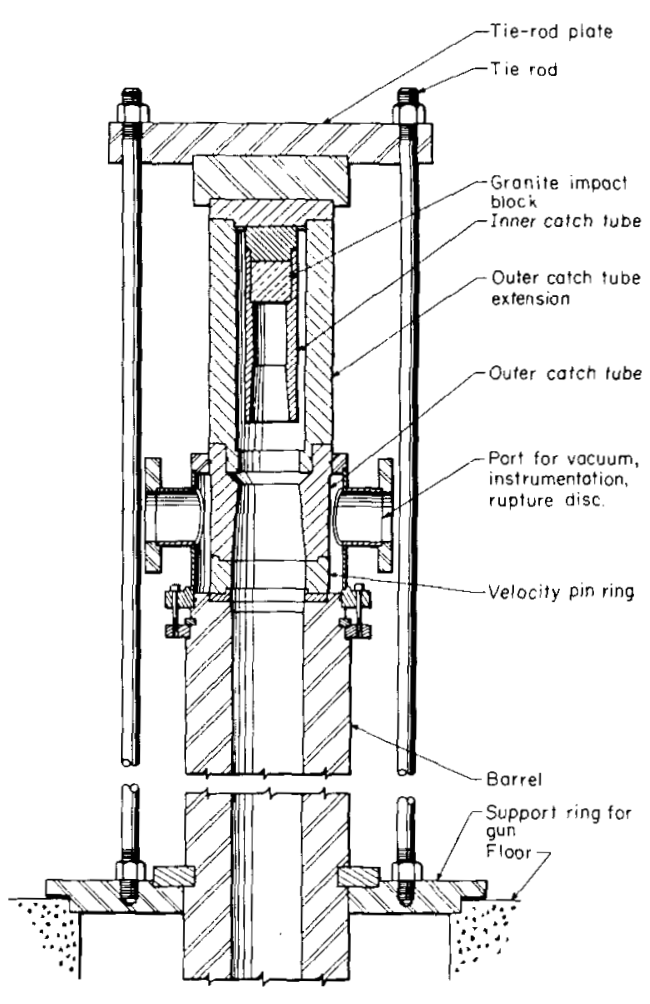

Fig. 2.

Standard impact chamber. 

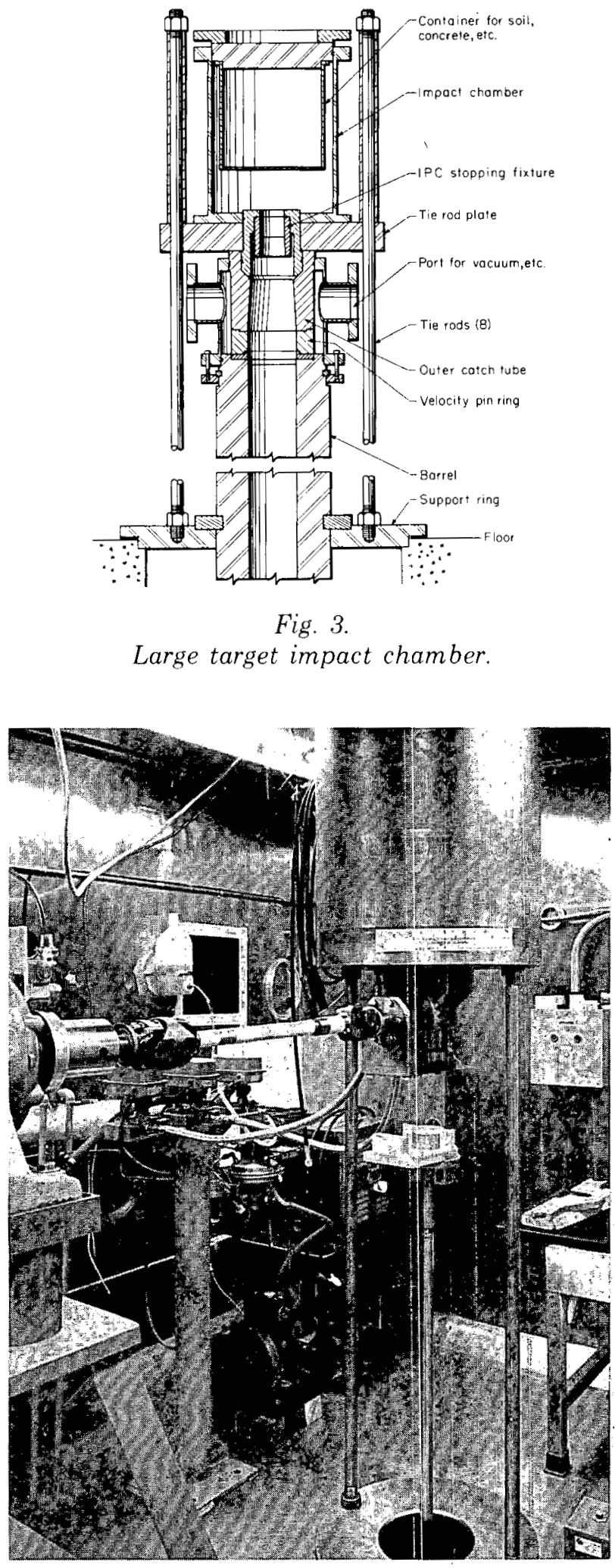

Fig. 4.

Gun breech.

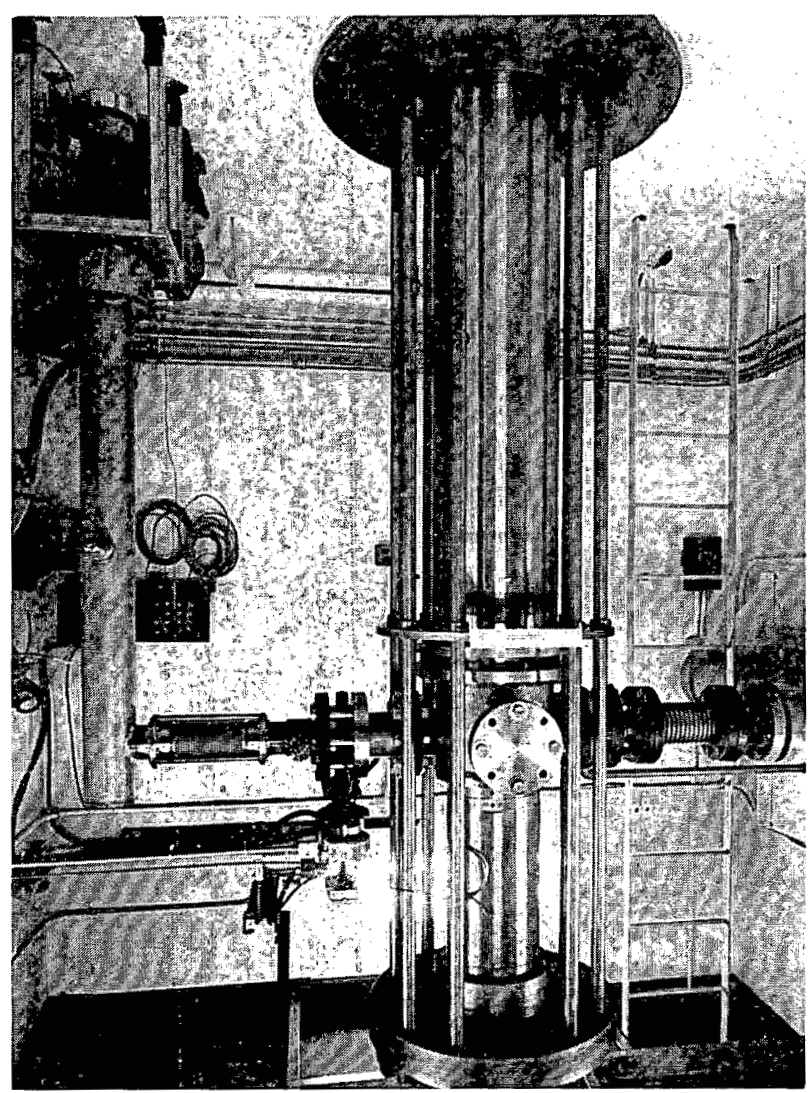

Fig. 5.

Standard impact chamber.

The gun fires vertically upward, extending to the upper floor level because of the length of the device. The upper room has forced ventilation with filtered intake air and triply filtered exhaust air. The forcedexhaust air from the room on the lower level is doubly filtered.

\section{A. Gun}

Previous experience at the Los Alamos Scientific Laboratory (LASL) demonstrated the capabilities of a single gas gun; i.e., that it can achieve projectile velocities from $<30 \mathrm{~m} / \mathrm{s}$ to $>300 \mathrm{~m} / \mathrm{s}$. Typically, gas guns have smooth bores and relatively large breeches containing compressed gas for propelling the projectile. After surveying the current and projected needs of the Division of Space Nuclear Systems, U.S. Energy Research and Development Administration. we decided that a $178-\mathrm{mm}$-diam bore would accommodate an acceptable variety of samples. Initial calculations indicated that a $10-\mathrm{kg}$ projectile could be accelerated with nitrogen propulsion gas to $\sim 330$ 


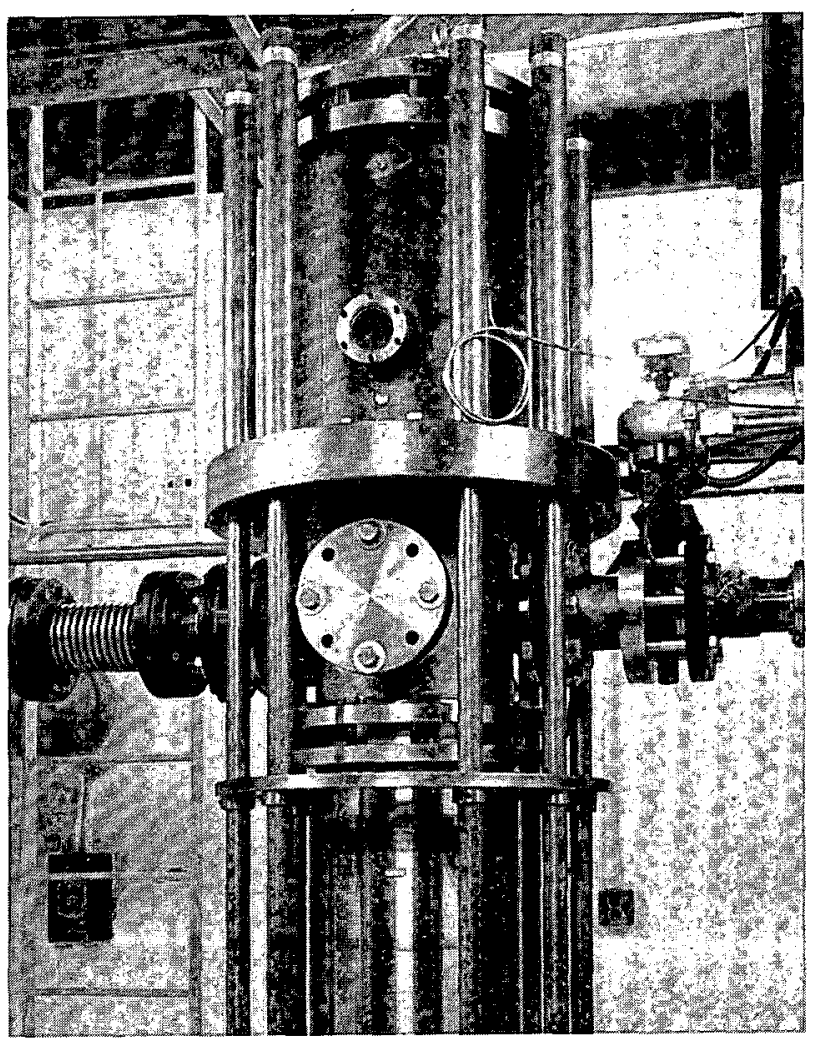

Fig. 6 .

Large target impact chamber.

$\mathrm{m} / \mathrm{s}$ using a 3.66-m-long barrel with a breech pressure of $13.8 \mathrm{MPa}$ if the breech volume equaled the bore volume. The final design is a $178-\mathrm{mm}$-diam, 3.8-m-long, smooth-bore gun. The breech and barrel volumes are nearly equal. The gun fires vertically upward so that gravity holds the sample in place on top of the projectile. Tris feature eliminates mechanical complications in achieving a free release of the sample.

During operation, the gun bore ahead of the projectile is evacuated for several reasons. When impacting hazardous materials, the barrel and impact chamber should be a single, sealed system to block escape of hazardous material from the system; if the barrel were not evacuated, a means to contain the potentially contaminated gas ahead of the projectile would have to be devised, thus making the system more complex. Many of the impact samples require an inert gas or a high-vacuum atmosphere to prevent sample deterioration. One type of furnace used in the gun must have a high vacuum to operate (Sec. II. B-3). Predicting the velocity of the projectile is much easier if the barrel is evacuated. Finally, evacuating the bore prevents it from rusting.

\section{B. Projectile}

There are two basic projectile types. One strikes a stationary sample located near the end of the barrel. Because we have not used this projectile in IFIT, it will be discussed only briefly in a later section. The other projectile contains the sample and carries it to the target at the correct velocity, attitude, and temperature. The IFIT projectile is an integral part of the system for sealing the radioactive sample within the impact chamber (Sec. II. E). It consists of some or all of the following components: the outer projectile cylinder (OPC), the inner projectile cylinder (IPC), the furnace, and the specimen capsule. Figures 7 and 8 show a typical projectile with a high-temperature furnace.

1. Outer Projectile Cylinder. The OPC, a metal cylinder of nominal bore diameter, is directly propelled by the breech gas. O-rings provide a sliding seal between the OPC and the bore. Depending upon the desired impact temperature, the OPC may have interior passages through which chilled water is circulated. The OPC base has a threaded

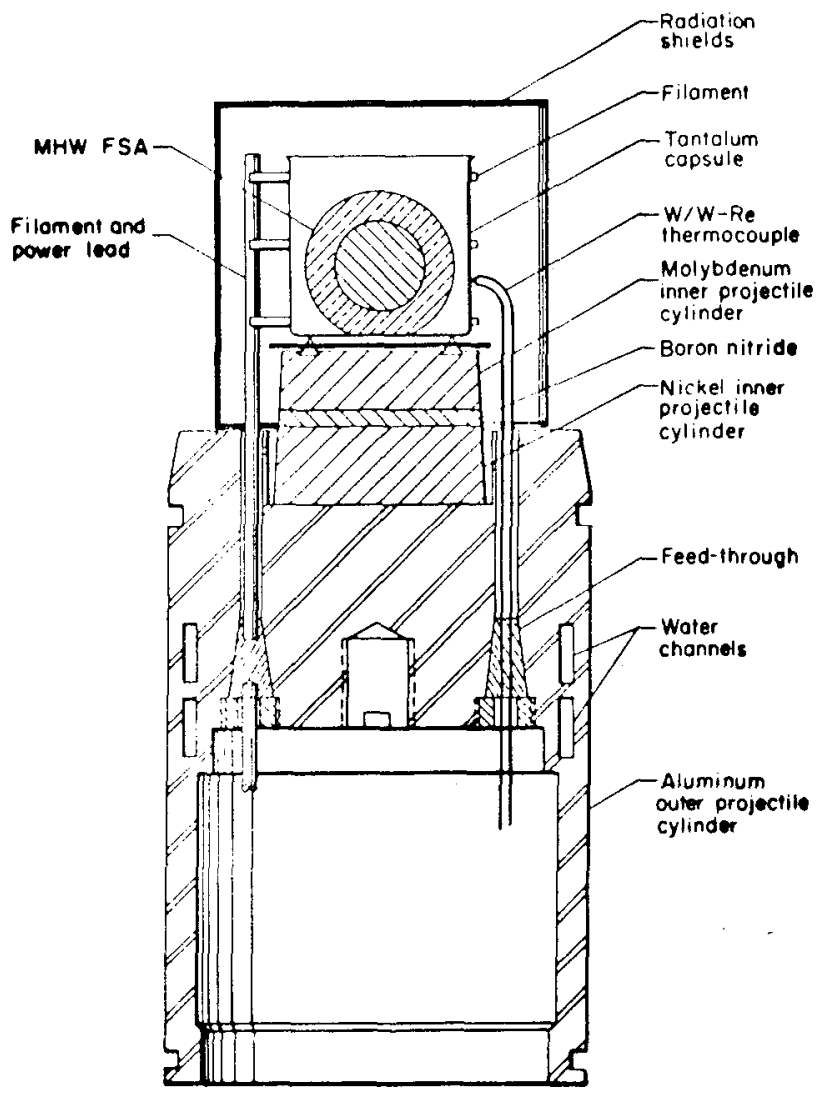

Fig. 7.

A typical projectile with an EB furnace. 


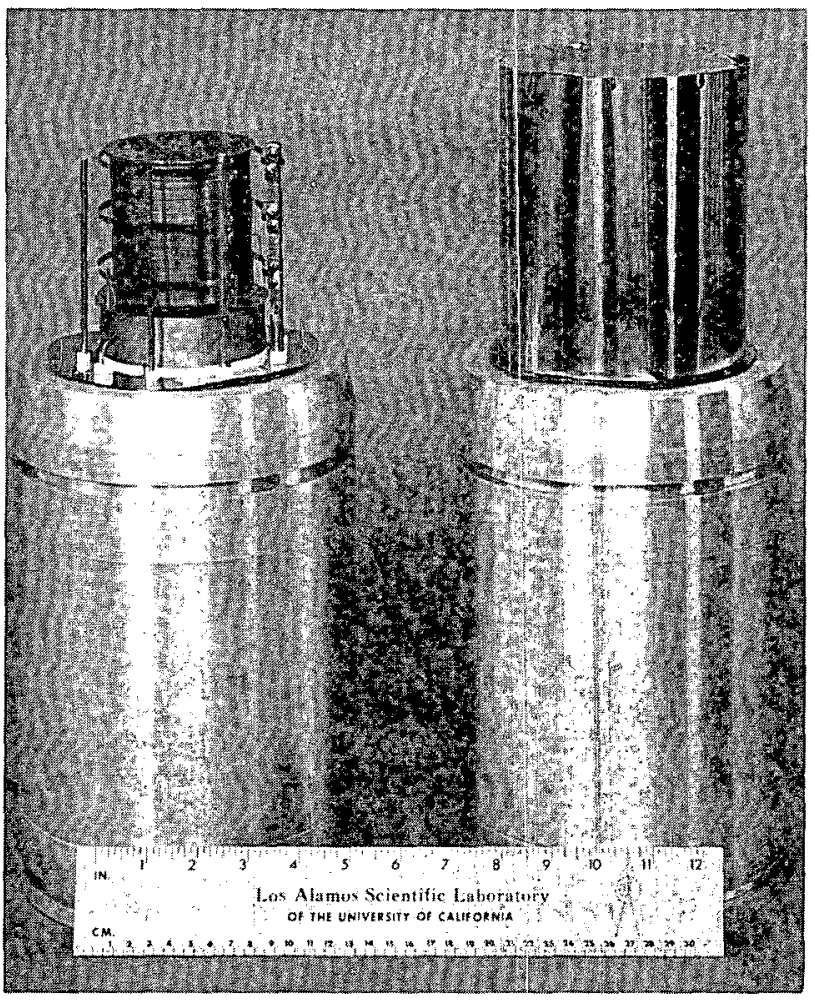

Fig. 8

Typical projectile with an EB furnace. Thermal radiation shield removed from left projectile to show furnace details.

hole that mates with the trigger (Sec. II. B). The $\mathrm{OPC}$ is held in the bore by the trigger until trigger actuation.

The OPC is usually made from aluminum, brass, or a composite of steel and aluminum, depending upon the planned impact velocity. Variations in the energy dissipated as barrel-OPC friction, even with approximately constant initial breech pressures and projectile masses, can cause variations in the impact velocities from shot to shot. However, when the projectile's kinetic energy is large compared to the friction loss, the variations of the friction losses will not cause significant variations in the impact velocities. Therefore, to achieve high kinetic energy at low velocities, the projectile must be massive, so highdensity materials (brass or the composites) are used to make the OPC. With high-velocity projectiles, high kinetic energies can be achieved with lessmassive projectiles and the OPC can be made of lowdensity materials such as aluminum or magnesium?

The OPC has several access holes through the base to route power and instrumentation leads from the breech to the furnace. Seals for these openings in the

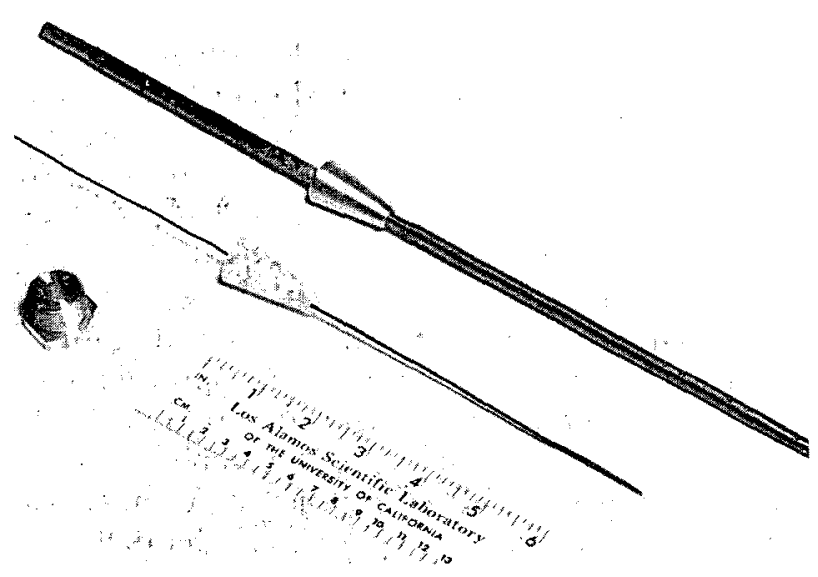

Fig. 9.

Electrical penetration seals and retainer nut for $O P C$.

OPC base must hold a high vacuum and pressure at all stages of gun operation. The high-vacuum and pressure-sealing capabilities are required while the projectile is in the barrel because of the propulsion gas pressure behind the projectile and the vacuum requirements in the barrel ahead of the projectile. Even after the projectile reaches the impact chamber, the electrical access seals in the OPC must remain intact to contain the plutonium, as described in Sec. II. E.

Two types of electrical-penetration seals are used in the OPC. The first is a truncated aluminum cone, with conducting rods screwed into each end (Figs. 7 and 9), and can conduct substantial current. Clear Glyptal varnish is applied to the cone surface for electrical insulation. A hole in the projectile base mates with the aluminum cone, providing a sealed electrical lead. Silastic is applied to the back edge of the aluminum cone as a back-up seal. A retainer nut, tightened against the aluminum cone, holds it in place. This electrical-penetration seal is rugged and is unaffected by the large accelerating forces and substantial deformations of the OPC.

The second type of seal is used for low-amperage applications. It is a high-durometer polyurethane truncated cone with conductors running through its center. A retainer nut also holds this cone in the $\mathrm{OPC}$. This penetration seal is also rugged and completely satisfactory.

2. Inner Projectile Cylinder. The IPC consists of a set of axially stacked, tapered cylinders, located in a recess in the face of the OPC (Fig. 7). The IPC 
provides the seal for the inner catch tube as described in Sec. II. E. Based on the desired impact temperature, various materials are used for the IPC. For a typical sample temperature of $1400^{\circ} \mathrm{C}$, the top cylinder of the IPC is made from molybdenum, and the bottom cylinder is made from nickel (Sec. II. D). A boron nitride insulator is inserted between the two metal cylinders. The top cylinder extends into the furnace and reaches a temperature approaching the furnace temperature; strength is required of this piece, so a refractory metal such as molybdenum is used. The lower cylinder is cooled by contact with the OPC; however, it is still moderately hot. Strength, ductility, and high thermal conductivity are required in this lower cylinder and nickel possesses these properties.

In several IFIT applications, a cradle was machined into the top of the IPC to orient the impact sample. With this modification, the sample stays in the proper orientation.

3. Projectile Furnace. The furnace is an integral part of the projectile because this permits heating the sample until the instant the gun is fired. Furnace power terminates when the projectile is propelled far enough to break the power leads running through the projectile's base.

Several different types of furnaces have been used. Electron-beam (EB) heating has been used for hightemperature applications but induction heating could be used. For lower temperatures, resistance heating and radiant heating have been used. Resistance heating is generally unsatisfactory for high temperatures because of the large power leads that are required. Also, the response of resistance elements to power change is relatively slow, a distinct disadvantage for certain heating cycles.

"Hevi-Duty" heating elements have been used in the resistance furnaces. Nickel or stainless steel thermal radiation shields are placed around the furnace; also, heat loss from the furnace is reduced because the furnace is in a vacuum.

$\mathrm{EB}$ heating is used for high-temperature applications because it is direct and efficient, using high voltage and low amperage. In a typical application (Figs. 7 and 8), the EB furnace has three filaments positioned coaxially with the capsule. These filaments are resistively heated to a temperature where they start to emit electrons spontaneously. A high positive voltage (200-2000 V, 0-7.5 A) is applied to the capsule to accelerate the emitted filament electrons into the capsule. When the electrons strike the capsule, their kinetic energy becomes thermal energy, producing direct and efficient heating of the capsule. Thermal insulation for the EB furnace consists of $0.025-\mathrm{mm}$ (0.001-in.) molybdenum radiation shields.

The EB furnace requires a vacuum to operate. With the configuration described here, arcing occurs between the filament and the capsule if the absolute pressure exceeds $\sim 60 \mathrm{mPa}$. The electrical potential across an arc is low and the amperage is high, which are characteristics directly opposed to EB heating and its power supply.

Sample temperatures of $1200-2000^{\circ} \mathrm{C}$ have been achieved with the typical EB furnace. The upper temperature limit is undetermined and is probably $>2000^{\circ} \mathrm{C}$. The sample heating rate is high with an EB furnace, a feature that has useful applications as described later.

So far, the only way that radiant heating has been used in the projectile is by using the $\mathrm{EB}$ furnace hardware in another mode. The filaments of the EB furnace are resistively heated to temperatures $>2000^{\circ} \mathrm{C}$, thereby providing a good radiant energy heat source. In this mode, the existing EB furnace is satisfactory for sample temperatures up to $\sim 1200^{\circ} \mathrm{C}$. Presently, hardware is being specifically developed for radiant heating.

4. Specimen Capsule. The impact sample is located on top of the IPC inside the furnace and usually has its own encapsulation as an integral part. However, a different sample capsule is required when the impact sample is radioactive and when the integral encapsulation does not provide positive radioactivity containment during the heating and accelerative conditions in the gun.

The EB furnace requires a target for electron bombardment. This may be the capsule containing the impact specimen or a thin cylindrical metal shield around the impact specimen. Direct electron bombardment of the impact specimen is normally not acceptable, primarily because of sample outgassing and the sample geometry.

The major capsule types are shown in Fig. 10. The capsule on the left has relatively thin walls and is assumed to fail when impacted. The capsule on the right has heavy walls and is designed to remain intact. Figure 11 shows the most commonly used capsule, which is formed by spinning $0.8-\mathrm{mm}$-thick tantalum sheet. It is $76 \mathrm{~mm}$ in diameter and $76 \mathrm{~mm}$ long. As shown in Fig. 11, a recess is formed in the bottom of the capsule to center the impact sample. Such capsules have been used at temperatures up to $2000^{\circ} \mathrm{C}$. 


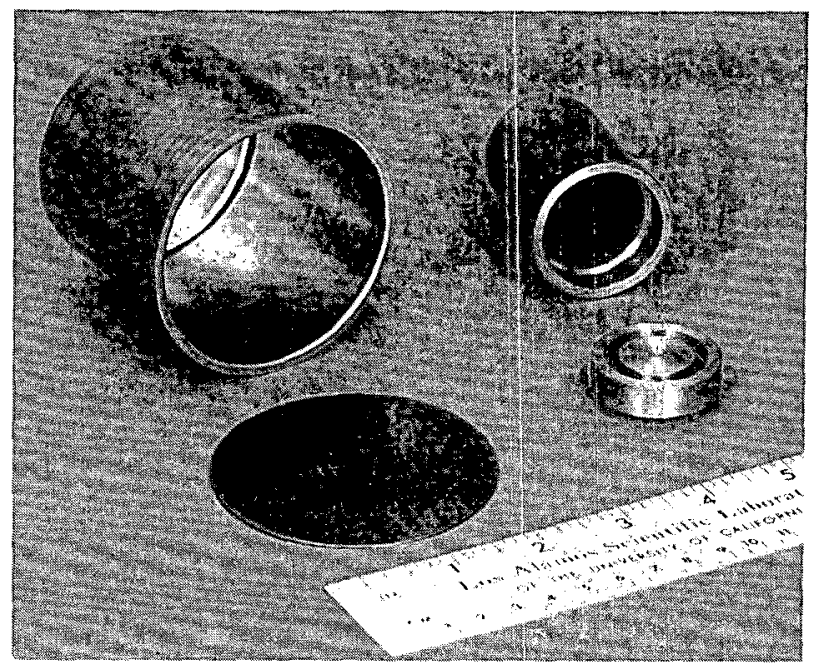

Fig. 10.

Two types of high-temperature tantalum impact capsules.

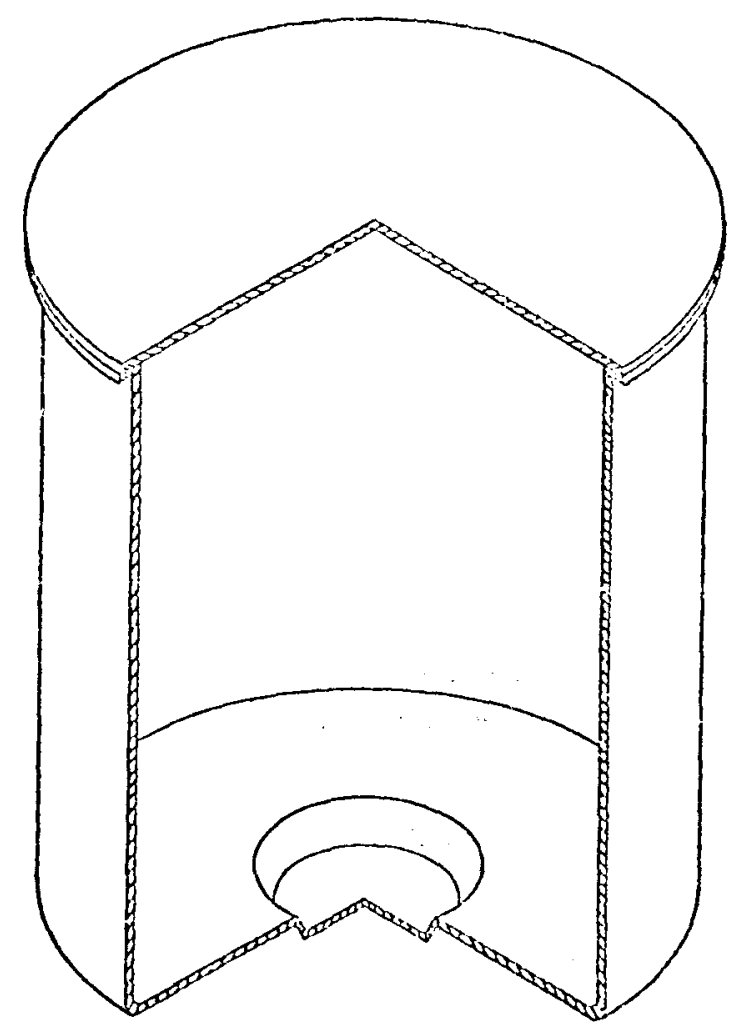

Fig. 11.

A thin-walled, high-temperature, tantalum impact capsule.
5. Furnace Temperature. The sample's temperature is measured by a thermocouple attached to the impact capsule or to another appropriate part of the projectile. A digital voltmeter and printer measure and record the thermocouple voltage. During $\mathrm{EB}$ furnace use, the capsule and thermocouple are at a high voltage $(200-2000 \mathrm{~V}$ ). To measure the thermocouple voltage when using the $\mathrm{EB}$, we electrically isolate the digital voltmeter from ground and from wall power with an isolation transformer; thus, the ground potential of the voltmeter is forced to float at the capsule potential, and the differential thermocouple voltage is read directly with the voltmeter.

\section{Trigger}

The OPC is held in place in the gun barrel by a triggering device that releases the projectile when ready to be propelled through the barrel. Three types of triggering devices are used, the choice depending on the test conditions. However, all three have the following common characteristics

- The breech is pressurized to shot pressure before projectile release.

- Release of the projectile will not occur until a large external mechanical force is applied to the triggering device (excluding major mechanical failure).

The standard trigger (Fig. 12) restrains the OPC by a threaded rod (trigger-screw rod) screwed into the base of the OPC. Concentric with this rod is a fixed thick-wall tube with two lugs that mate with slots in the OPC, preventing it from rotating as the trigger-screw rod is screwed out of the OPC during projectile release.

The interrupted-thread trigger (Fig. 13) is the same as the standard trigger except the trigger-screw rod has interrupted threads that mate with interrupted threads in the OPC. This trigger releases the projectile when the trigger-screw rod has rotated $\sim 120^{\circ}$. Thus, projectile release can be more easily synchronized with certain types of equipment, such as high-speed movie cameras.

The hydraulic trigger (Figs. 13 and 14) is used at higher breech pressures, where the torque required to turn a trigger-screw rod is too high. The heart of this trigger is the break rod, which is threaded at one end and has a cavity in the other end. The threaded end of the break rod screws into the base of the OPC. A section of the break rod is reduced in outside diameter so that sufficient strength remains to hold 


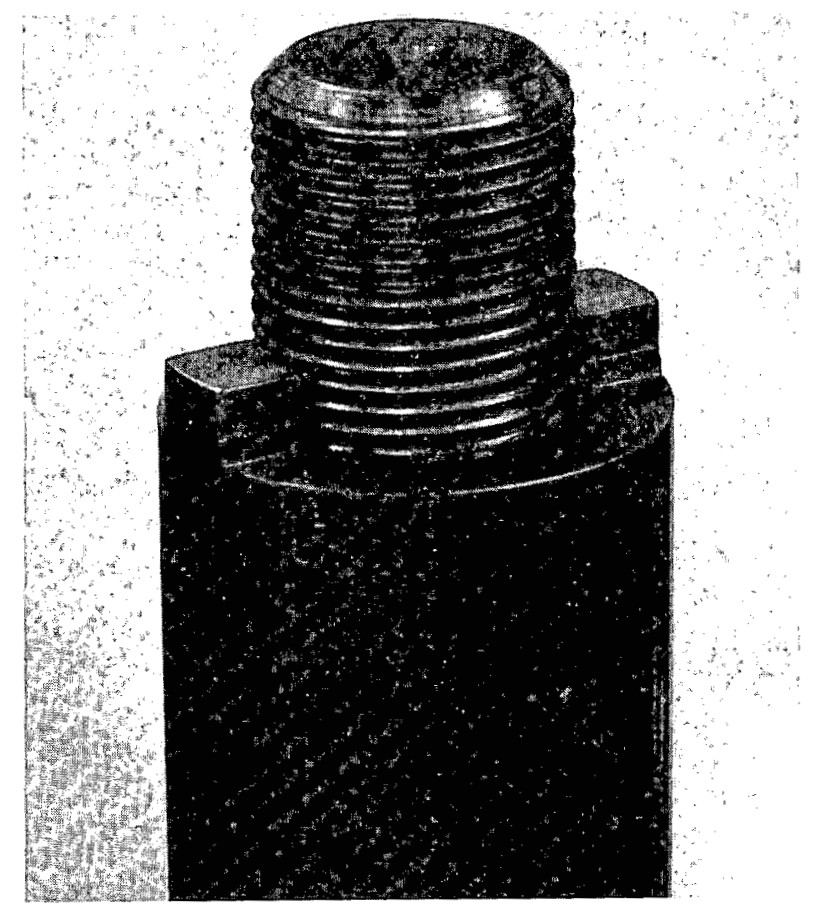

Fig. 12 .

Standard screw trigger.

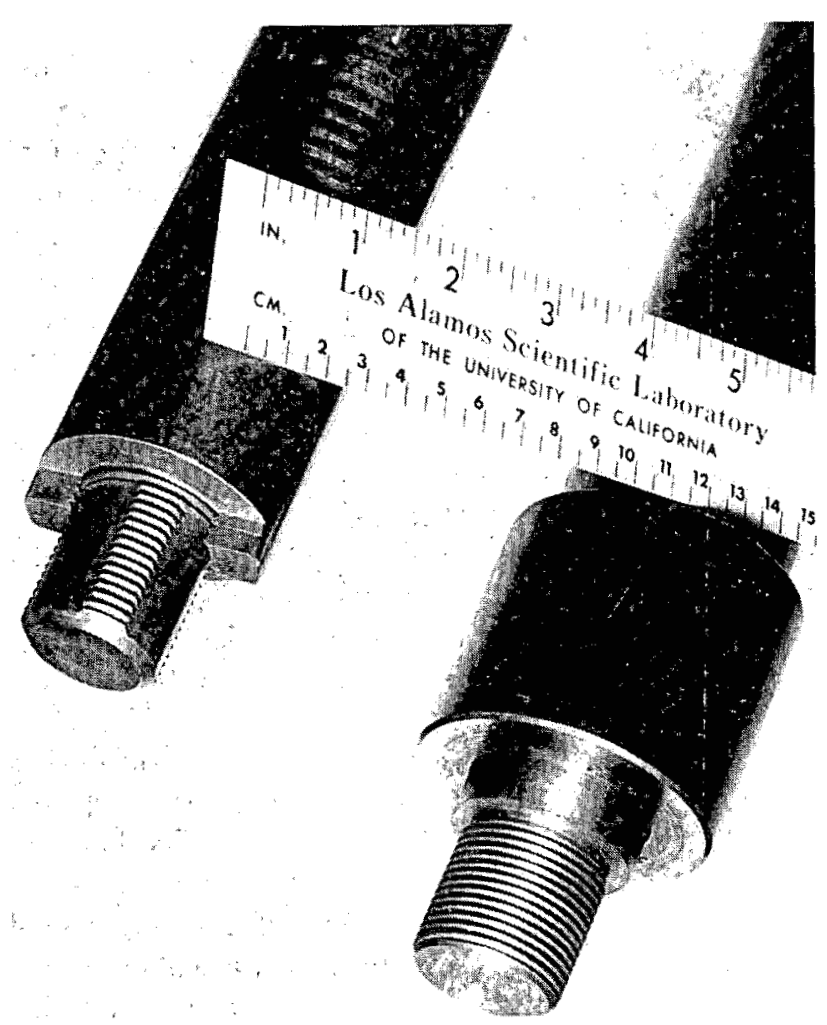

Fig. 13.

Interrupted-thread trigger and hydraulic trigger.

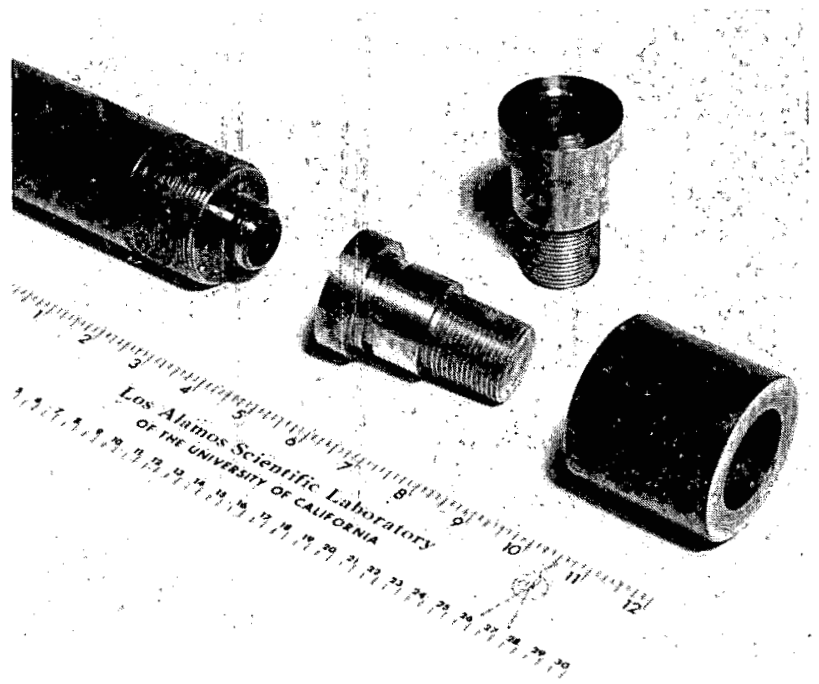

Fig. 14.

Hydraulic trigger components.

the projectile when the breech is pressurized. About $27 \mathrm{kN}$ of force above the breech pressure is required to break the rod. To release the projectile, sufficient hydraulic pressure is applied in the break-rod cavity to break the rod at the reduced section, releasing the projectile. Hydraulic pressures up to $138 \mathrm{MPa}$ can be applied to the break rod.

\section{Determination of Impact Velocity.}

Impact velocity is determined by measuring the OPC velocity. Situated between the impact chamber and the muzzle is a velocity pin ring, a fixture holding 10 pairs of active and grounded pins spaced at known increments along the centerline of the gun (Fig. 15). The active pins are held at a small electrical potential above ground potential. When the top of the OPC strikes an active pin, the pin is shorted to ground through the OPC and a ground pin. Each shorted pin initiates a voltage pulse that is displayed and recorded on a raster oscilloscope. Time markers are mixed with the sweep of the oscilloscope for time calibration (Fig. 16). Knowing the position of each pin and the time when each pin is struck by the OPC. the velocity of the projectile assembly can be calculated. A linear least squares fit is used to calculate the velocity, with a typical standard deviation of $\pm 0.2-0.5 \%$. 


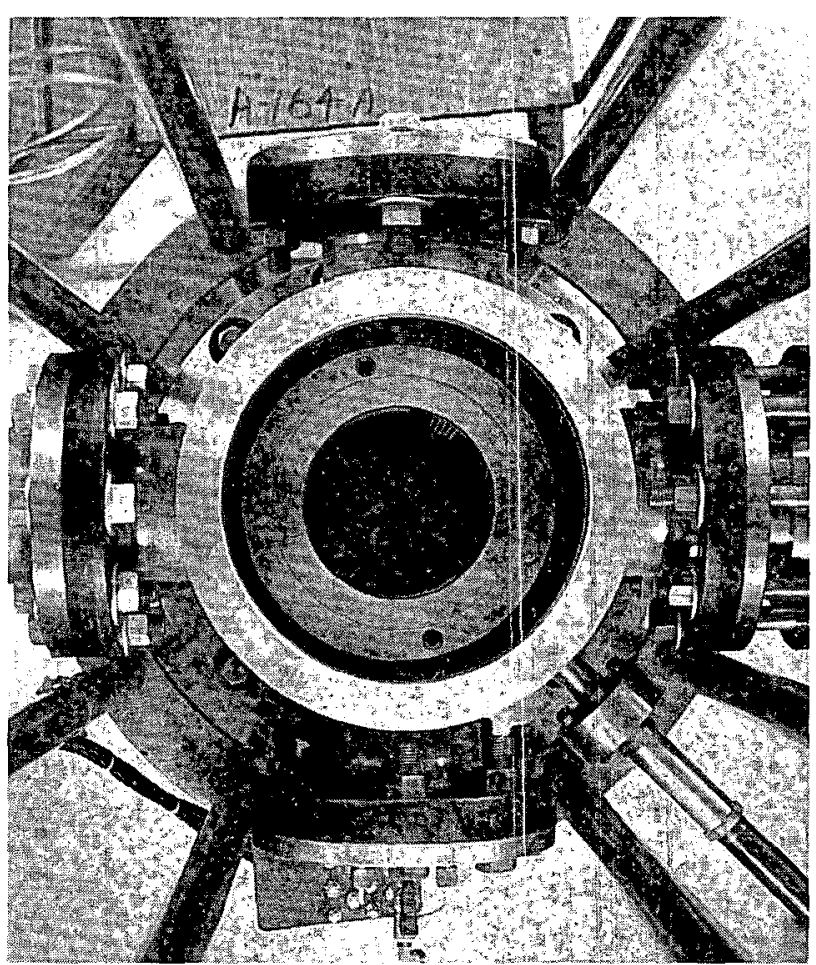

Fig. 15

Velocity pin ring on top of gun barrel. (View looking downward into muzzle).

\section{E. Impact Chamber}

The impact chamber is sealed to the gun muzzle and contains the impact target or impact specimen. The preferred IFIT mode is to have a fixed target with the impact sample accelerated by the projectile. The reason is that the sample velocity is low after impact and additional damage to the impact sample from secondary impacts at rebound velocity is usually insignificant. If the projectile is the target, secondary impacts of the sample can be very damaging unless proper precautions cire taken; this is

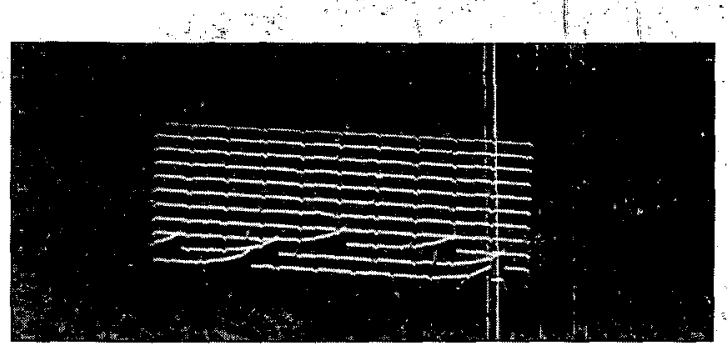

Fig. 16 .

Raster oscilloscope record of velocity pin data. Small pulses on the sweep are time markers; large pulses are velocity pin contact pulses. because the sample velocity is high after the primary impact, i.e., the sum of the impact and rebound velocities of the sample relative to the projectile. Discussion in this section will be directed primarily towards the fixed target mode of operation.

Besides being the impact region, the impact chamber confines all impacted hazardous materials in a sealed vessel and isolates parts of the projectile from the impact. By confining the impact components, the impact chamber protects the rest of the facility from impact-generated debris. To provide the confinement and isolation functions, a catch tube developed by another laboratory, ${ }^{2}$ was incorporated into the impact chamber. The catch tube is a heavy-walled tube concentric with the centerline of the gun and having a female taper with the large end toward the muzzle. The length and angle of the taper depend upon the application. The catch tube stops the IPC or the OPC by having the projectile run into and jam in the catch-tube taper. forming a tight seal at the end of the catch tube.

The two basic types of impact chambers that have been developed are the standard impact chamber and the large target impact chamber. These impact chambers are described in the following sections. Means of "watching" the impacts in either type of impact chamber are also described.

1. Standard Impact Chamber. The standard impact chamber consists of an outer catch tube (OCT) assembly and an inner catch tube (ICT) assembly. Figures 2 and 5 show the standard impact chamber. The typical OCT assembly consists of an OCT (Fig. 17), an OCT extension, and an OCT end plate. The assembly is shown in Fig. 18. When the gun is fired, the OPC jams into the OCT to form a sealed vessel containing everything riding on top of the OPC. The IPC and the capsule containing the impact sample go into flight within the $\mathrm{OCT}$ assembly. Forces required to dislodge the OPC from the OCT are typically 1.4-14 GPa ( 100 to 1000 tons). The OCT is reusable and is recovered after each impact by pressing the OPC out of the OCT.

The typical ICT assembly (Fig. 19) consists of an ICT, an ICT end plate, and a target. When the capsule containing the impact sample and the IPC enter the ICT, the capsule strikes the target, and the IPC jams into the taper of the ICT, forming a second sealed vessel that then becomes the primary sealed vessel. Forces required to dislodge the IPC from the ICT are typically $\geq 0.7 \mathrm{GPa}$ (50 tons). Usually, the ICT is not reusable.

The sample has double containment inside the two sealed catch tube assemblies. This arrangement 


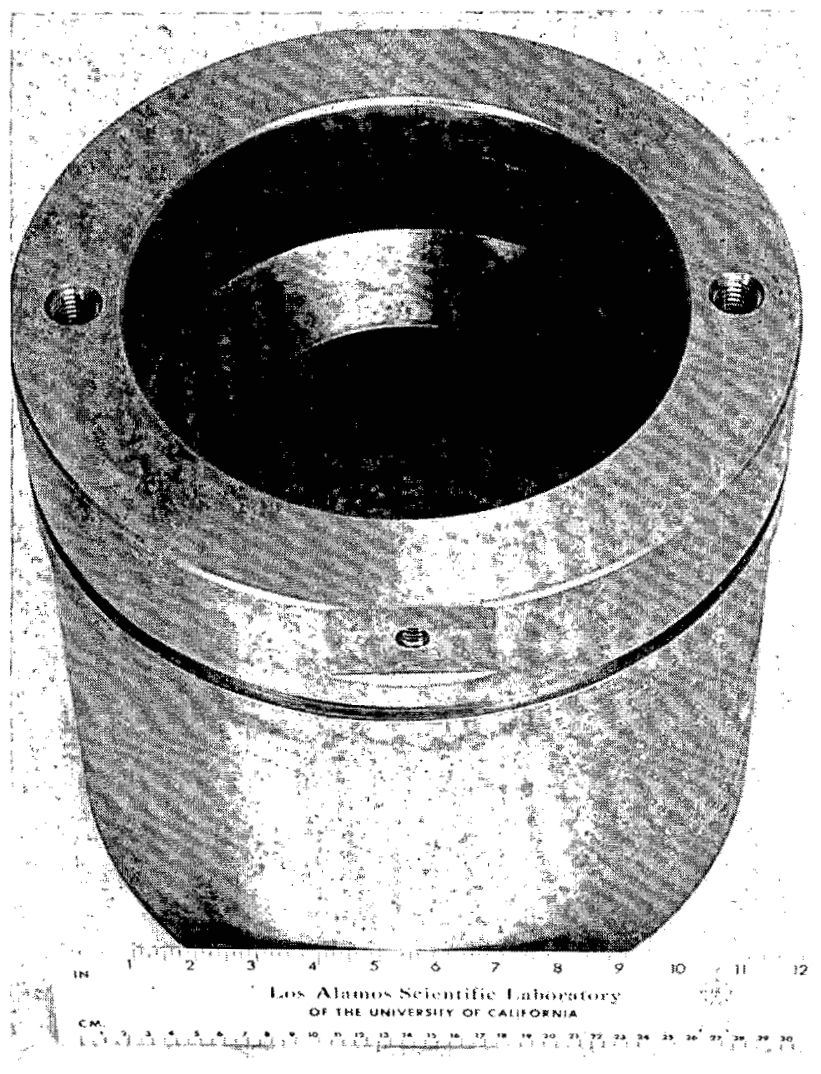

Fig. 17.

Outer catch tube.

was developed to contain impacts safely when testing very hazardous materials such as ${ }^{238} \mathrm{PuO}_{2}$.

Examples of targets used in the IC $\mathrm{T}$ are blocks of granite and steel and shaped targets such as small angle irons. Any object capable of being fixed inside the ICT can be used as a target. Shown in Fig. 19 is a granite cylinder that is often used as a standard impact target. Usually the granite blocks are $102 \mathrm{~mm}$ in diameter by $102 \mathrm{~mm}$ long and have a $0.813-\mu \mathrm{m} \mathrm{(32-}$ $\mu$ in.) rms surface finish.

The IPC is often at high temperature when it jams into the ICT. When it cools, the difference in thermal contraction of the IPC and the ICT may be enough to compromise the catch-tube seal. To compensate for this, the tapered section of the ICT is wrapped with a heating tape and preheated to $150^{\circ}$ $200^{\circ} \mathrm{C}$. This is adequate compensation where the steady-state temperature of the projectile furnace is $\leq 1400^{\circ} \mathrm{C}$.

When the impact sample contains hazardous materials, it is recovered by bagging and removing the ICT assembly from the OCT assembly. After

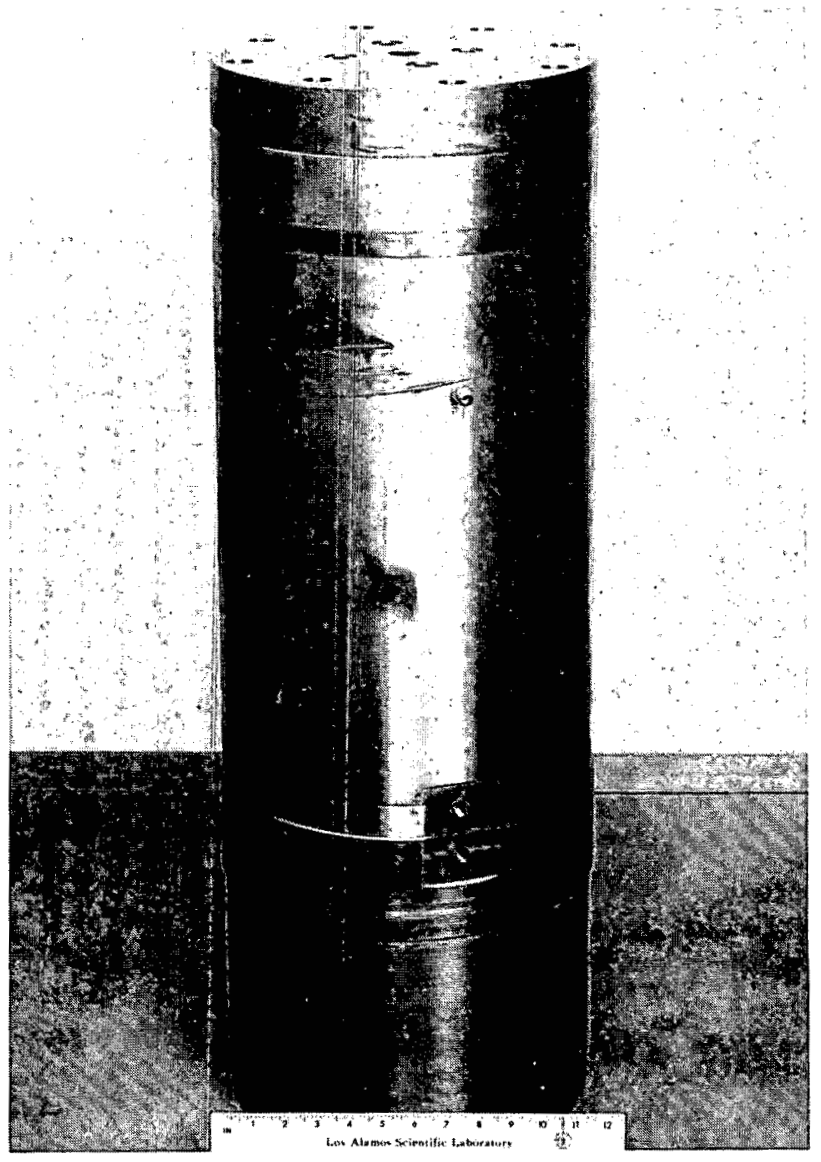

Fig. 18.

Outer catch tube assembly.

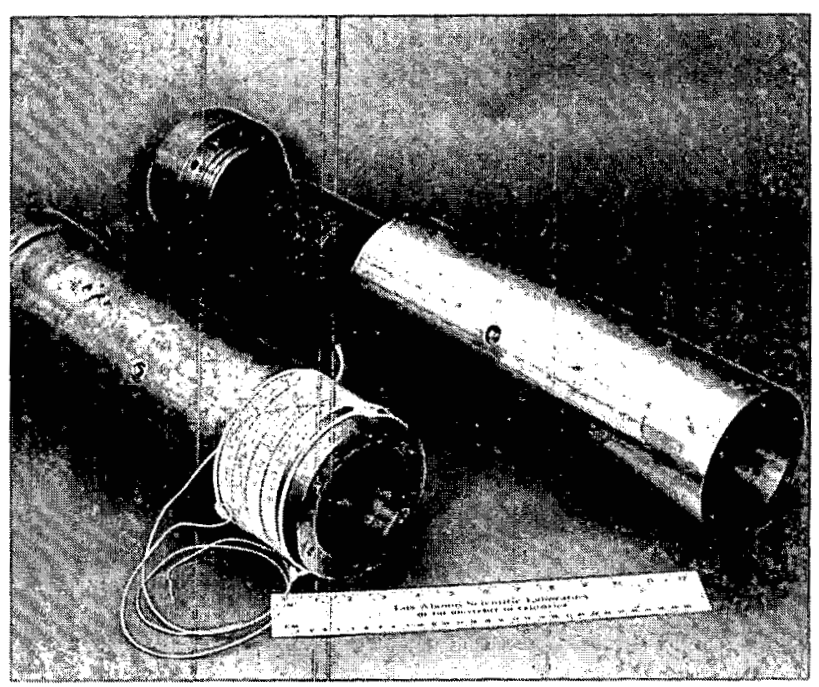

Fig. 19.

Standard inner catch tube assembly, assembled and disassembled. 
checking the exterior surfaces of the ICT assembly, it is disassembled in a glovebox train and the specimen is recovered.

Because impacts involving hazardous materials require that the ICT assembly be disassembled in a glovebox train, the size and weight of the assembly cannot exceed certain limits, or it would become unmanageable. However, we receive requests to do impacts against targets that are large compared to the standard size target. To meet these needs a special ICT assembly was developed. It is shown in Figs. 20 and 21. The catch tube is more complex than the standard one, consisting of an ICT, a retainer ring, a target chamber, and an ICT end plate. Its unique feature is that the impact block and the catch tube, in which the impact occurs, are separate chambers divided by a $0.8-\mathrm{mm}$ stainless steel membrane welded to the ICT. To minimize the membrane's effect, it is either in or nearly in contact with the impact target, which must be a fairly rigid and flat. Proper functioning of the assembly requires that the stainless steel membrane not be ruptured by the impact. The target chamber and the ICT can then be safely separated after impact, and the smaller ICT can be taken to a glovebox train to be opened.

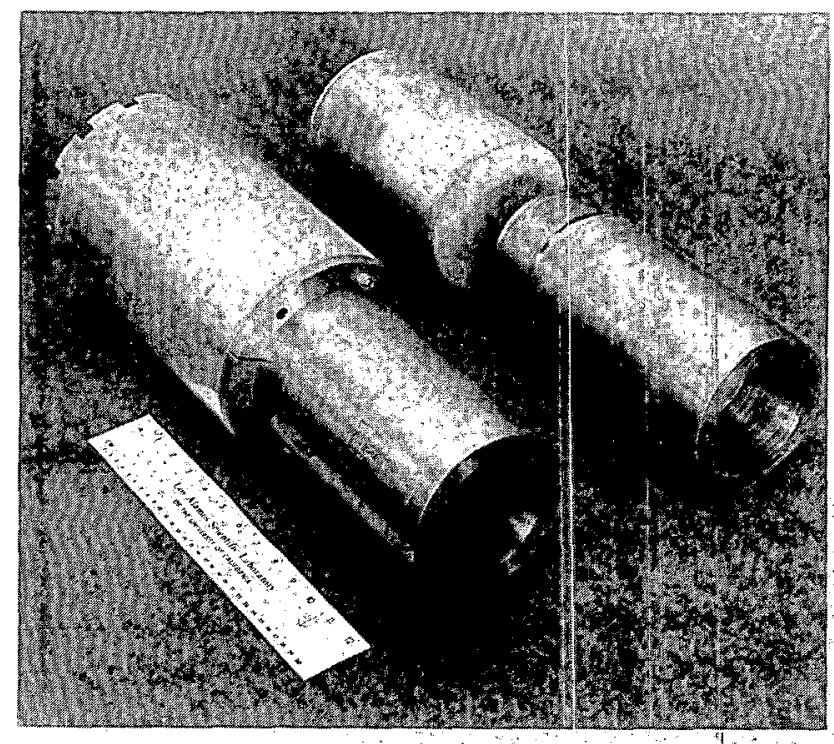

Fig. 20.

Inner catch tube assembly for concrete target impacts in standard impact chamber. Parts on left are assembled and parts on right are concrete target and inner catch tube before assembly.

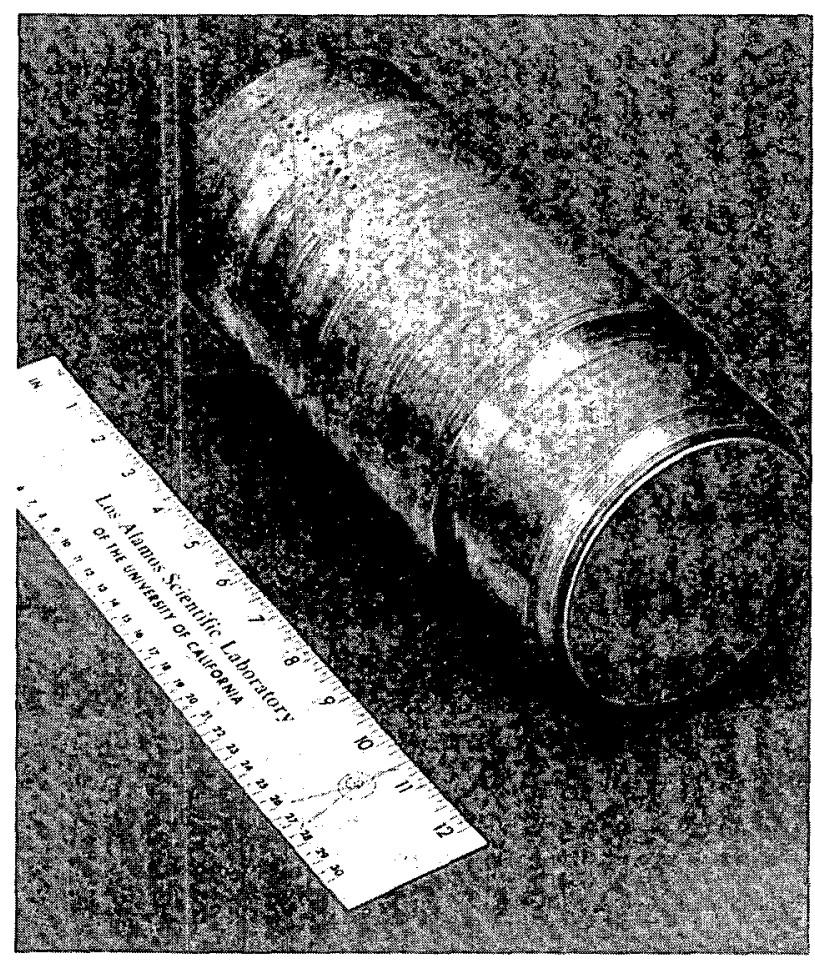

Fig. 21.

Inner catch tube for concrete target impacts showing stainless steel membrane.

Another desirable feature of this ICT assembly is that the target is isolated from the gun, so that targets with bad outgassing characteristics (such as concrete) do not affect the vacuum within the gun.

Using existing OCT extensions, the size of a concrete block that can be fitted into the OCT extension is $140 \mathrm{~mm}$ in diameter by $200 \mathrm{~mm}$ long. However, experience indicates that this scheme might be adapted to the large target impact chamber described in the next section.

2. Large Target Impact Chamber. Impetus for developing the large target impact chamber came from a request to do impacts against soil. To retain the compressibility and flow characteristics of soil, the volume of the target must be larger than could be handled in the standard impact chamber. Also, we must use other large targets such as concrete and certain shaped targets. Presently, the large target impact chamber cannot be used for hazardous materials.

A drawing and a photograph of the large target impact chamber are shown in Figs. 3 and 6 , respectively. Basic components of the impact chamber are the standard OCT, the ICT which does not form a sealed 
chamber, ${ }^{*}$ and an impact vessel. Inside the impact vessel there may be another vessel, the target vessel, containing targets such as soil and concrete. This vessel has a hole in its bottom that alines with the gun muzzle. The impact sample strikes the target through this hole. A polyethylene sheet seals this hole so that a separate vacuum system can be used on the target vessel when the target has bad outgassing characteristics, such as soil possesses.

Targets used without the target vessel include steel shapes. The steel impact targets are either large flat plates or structural shapes, such as a "T" oriented so that the impact occurs against the end of its leg.

3. Photographing Impacts. High-speed photography has been developed for both classes of impact chambers. Photography has been used with impacts involving materials of low hazard levels. Photographing impacts of hazardous materials is probably possible, albeit expensive.

Let us first consider photographing impacts using the standard impact chamber. The OCT extension differs from the standard OCT extension in that it has a viewing window (Fig. 22) built into it. The window is made of polycarbonate, which is resistant to breakage. The modified OCT end plate has vacuumtight electrical feedthroughs for lighting power and a means to attach a lighting fixture and an impact blick (Fig. 23), which is usually made of steel. Four quartz halogen lights, two at $90^{\circ}$ and two at $45^{\circ}$ to the line of sight, are mounted in the lighting fixture. Either 650 - or $1000-\mathrm{W}$ lights are used. Because no ICT is used in this arrangement, no impacts involving hazardous materials can be done. In principle, an ICT assembly could be built with inside lights and with a window alined with the window in the OCT assembly so that the impact could be photographed. Such an ICT assembly would be more expensive because of the higher cost of a complex ICT assembly that could be used only once. Also,

* Because the impacted sample in this setup is not in a sealed vessel that can be taken to a glovebox train for sample recovery, the large target impact chamber is not suitable for impacting hazardous materials. We think that the modified ICT assembly described above could be adapted to the large target impact chamber so that the ICT would be a sealed chamber after the impact, one end of the ICT being sealed by the IPC and the other by a stainless steel membrane. Restrictions on this system would be that the target must be fairly rigid and flat and that the top end of the ICT must be in contact, or nearly in contact, with the target.

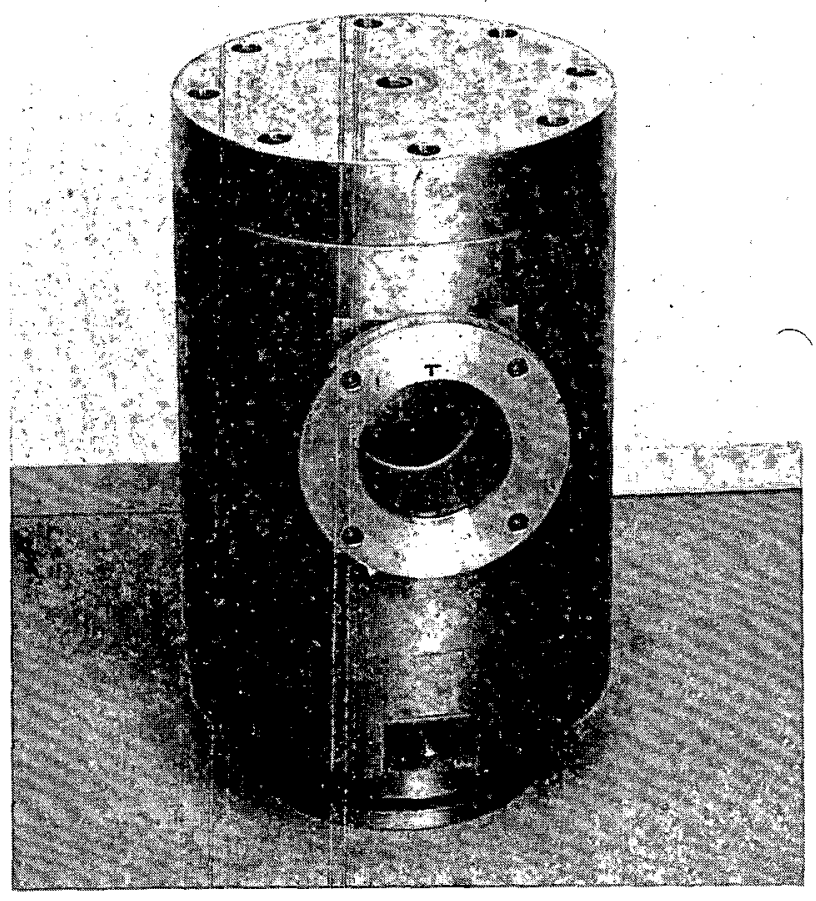

Fig. 22.

Outer catch tube extension for high-speed photography.

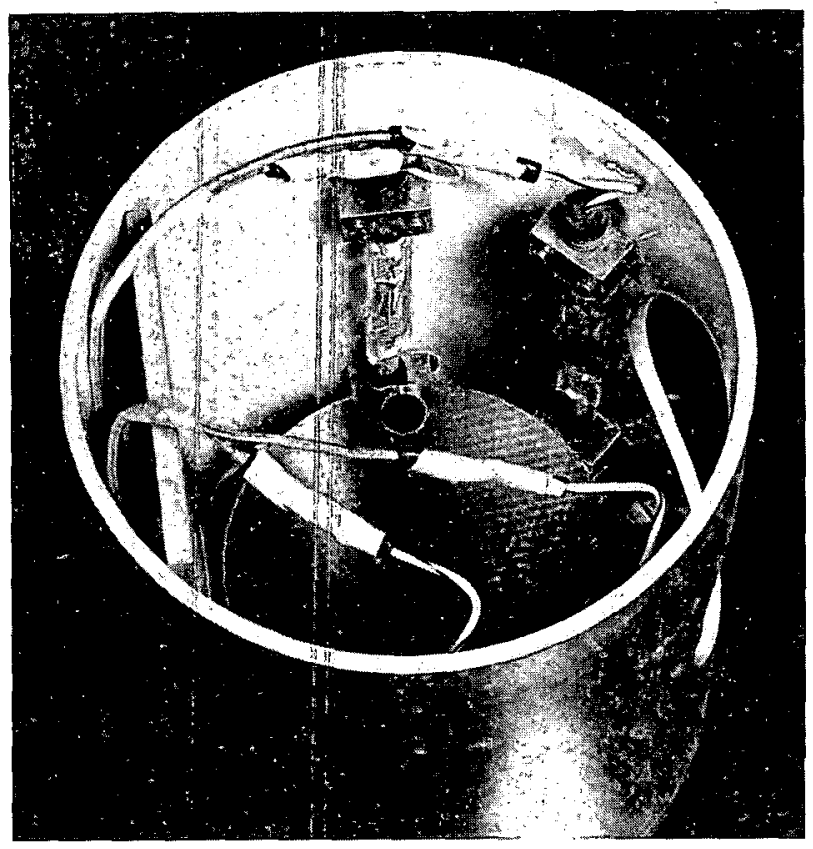

Fig. 23.

Lighting fixture and impact target attached to outer catch tube end plate. 
certifying that the windows would not be broken by the impact is difficult, and certification is necessary for hazardous materials.

We modified the large target impact chamber for photography by installing the same lighting arrangement, a similar lighting fixture, and a viewing window as that used in the standard impact chamber.

A Hycam Camera records the impacts. Its maximum framing rate is $\sim 9000$ frames/s. A half-frame head and a quarter-frame head are also available for the camera, providing framing rates of 18000 halfframe pictures/s and 36000 quarter-frame pictures/s, respectively. About $30 \mathrm{~m}$ of film must be run through the camera before it approaches its maximum framing rate, which is maintained for $1-2 \mathrm{~s}$, i.e., until the end of the reel is reached. Consequently, release of the projectile must be carefully synchronized with the camera. To synchronize the camera and gun, a microswitch in the camera is actuated when $\sim 30 \mathrm{~m}$ of film has passed through the camera. The microswitch closure initiates a trigger signal, actuating the interrupted-thread trigger. The trigger-release time is short enough to obtain pictures of the impact on the rest of the film.

4. Radiographs of Impacts. The same impact chambers used for photography can be used to obtain one flash $\mathrm{x}$-ray radiograph of an impact. However, because light is not required for radiographs, a metal window can be substituted for the polycarbonate window. This can be a significant safety factor compared to high-speed photography of impacting hazardous materials.

\section{F. Energy Absorption System}

Large reaction masses of steel in various components are located on top of the OCT. Thus, most of the kinetic energy of the projectile is dissipated by plastic deformation of the various components of the impact assembly. The remaining (residual) kinetic energy of the projectile is transferred into kinetic energy in the reaction mass and is absorbed by stretching tie rods (Figs. 5 and 6) connected to the reaction mass. The tie rods are sized so that they are not stretched beyond their limit. Less than $5 \%$ of the kinetic energy of the projectile is absorbed by the tie rods.

Components undergoing significant plastic deformation when stopping the projectile are the OPC, the IPC, and the ICT. Other components are sized to resist plastic deformation.

\section{APPLICATIONS}

Various types of impact tests that have been run in the IFIT are presented in this section. These examples do not define IFIT's limits, but do give some indication of its capabilities. All instances involve impact testing of heat sources for RTGs. Most tests were run using heat sources fueled with ${ }^{238} \mathrm{PuO}_{2}$, but some were performed with $\mathrm{ThO}_{2}$ or $\mathrm{UO}_{2}$ as simulant fuels. These tests principally determined whether the heat sources could withstand impacts under specified temperature and velocity conditions. Because the heat sources were being tested near their failure limits, the IFIT features insuring total containment of any hazardous material were necessary.

\section{A. Multihundred Watt System}

The Multihundred Watt (MHW) system is a thermoelectric power supply using a ${ }^{238} \mathrm{PuO}_{2}$ radioisotope heat source. ${ }^{3}$ Power from the $\mathrm{MHW}$ system is used to run electronic equipment on orbital satellites or interplanetary spacecraft. IFIT has tested the response of the heat source under a variety of postulated conditions, where either the heat source reenters the Earth's atmosphere and strikes the ground or a rocket launch-pad accident causes the heat source to drop to the ground. When the heat source reenters the Earth's atmosphere, it is rapidly heated and cooled by passing through the atmoshpere at high to moderate velocities. This heat cycle is simulated in the gun and is followed immediately by the impact, as in the actual case.

The MHW heat source is built as a modular assembly of 24 individually encapsulated $100-\mathrm{Wt}^{*}$ heat sources. Each source consists of a ${ }^{238} \mathrm{PuO}_{2}$ sphere inside a $0.51-\mathrm{mm}$-thick by $40.6-\mathrm{mm}-\mathrm{o} . \mathrm{d}$. iridium capsule that is surrounded by a spherical graphite-carbon composite impact shell with an $11.7-\mathrm{mm}$-thick wall. The assembled MHW heat source is too large to be impacted in IFIT. Usually, only one MHW sphere at a time is tested in IFIT, but a few double-sphere impacts were done with one sphere stacked on top of the other.

For a typical MHW sphere impact, the sphere is first taken through a reentry temperature pulse (Fig. 24). The EB furnace is used for the reentry temperature pulse. As the reentry pulse ends, the projectile is released, and the sphere strikes a granite

*Wt - watt thermal. 


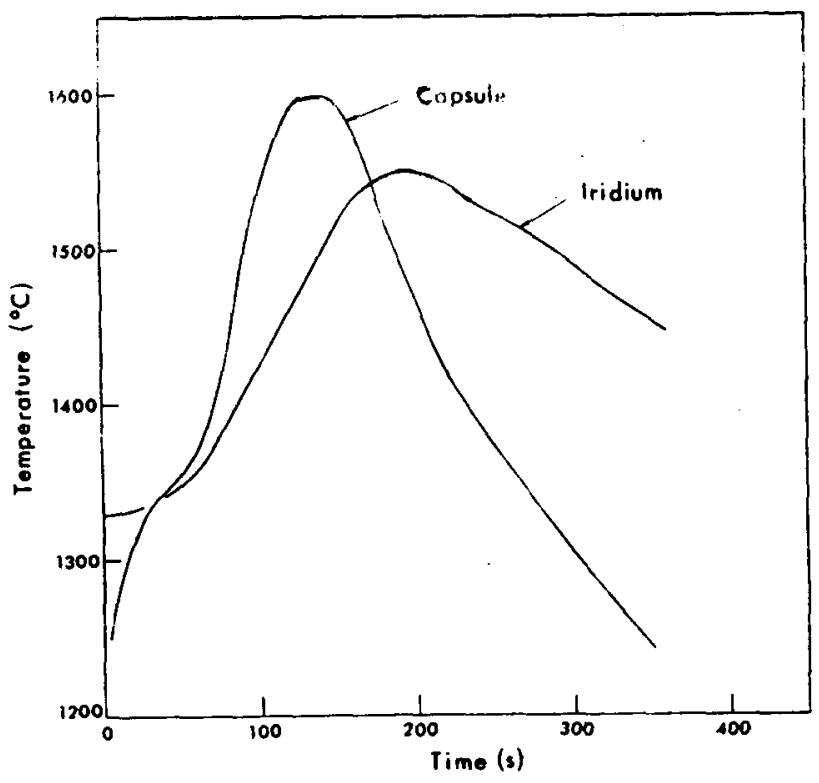

Fig. 24.

Reentry temperature pulse curve showing tantalum impact capsule temperature and calculated iridium temperature.

target at $85-87 \mathrm{~m} / \mathrm{s}$. Figure 25 shows an impacted MHW sphere before and after half of the impact capsule was removed from the MHW sphere.

MHW spheres fueled with ${ }^{238} \mathrm{PuO}_{2}$ were impacted against granite in the standard impact chamber, except for one sphere that was impacted against concrete with the special ICT assembly. Impact velocities were $75-87 \mathrm{~m} / \mathrm{s}$, and the impact temperatures of the iridium cladding were 1100 $1550^{\circ} \mathrm{C}$. With few exceptions, these spheres were impacted in sealed tantalum capsules like those shown in Fig. 11 .

MHW spheres with $\mathrm{ThO}_{2}$ simulant fuel were impacted against granite, concrete, soil, and structural steel shapes in either the standard or the large target impact chamber. Impact velocities were 29 to $87 \mathrm{~m} / \mathrm{s}$, and impact temperatures were typically $1100-1440^{\circ} \mathrm{C}$.

\section{B. Viking}

Viking refers to an RTG being used in the Viking mission to Mars. ${ }^{4}$ The RTG heat source uses a ${ }^{238} \mathrm{PuO}_{2}$ - $\mathrm{Mo}$ cermet and is cylindrical in shape with hemispherical ends. It is $52.5-\mathrm{mm}$ in diameter and is $127.3-\mathrm{mm}$ long. Because of its length and because of the lack of suitably large impact chambers at the time of the tests, only end-on impacts could be done in IFIT. The impacted heat sources contained a
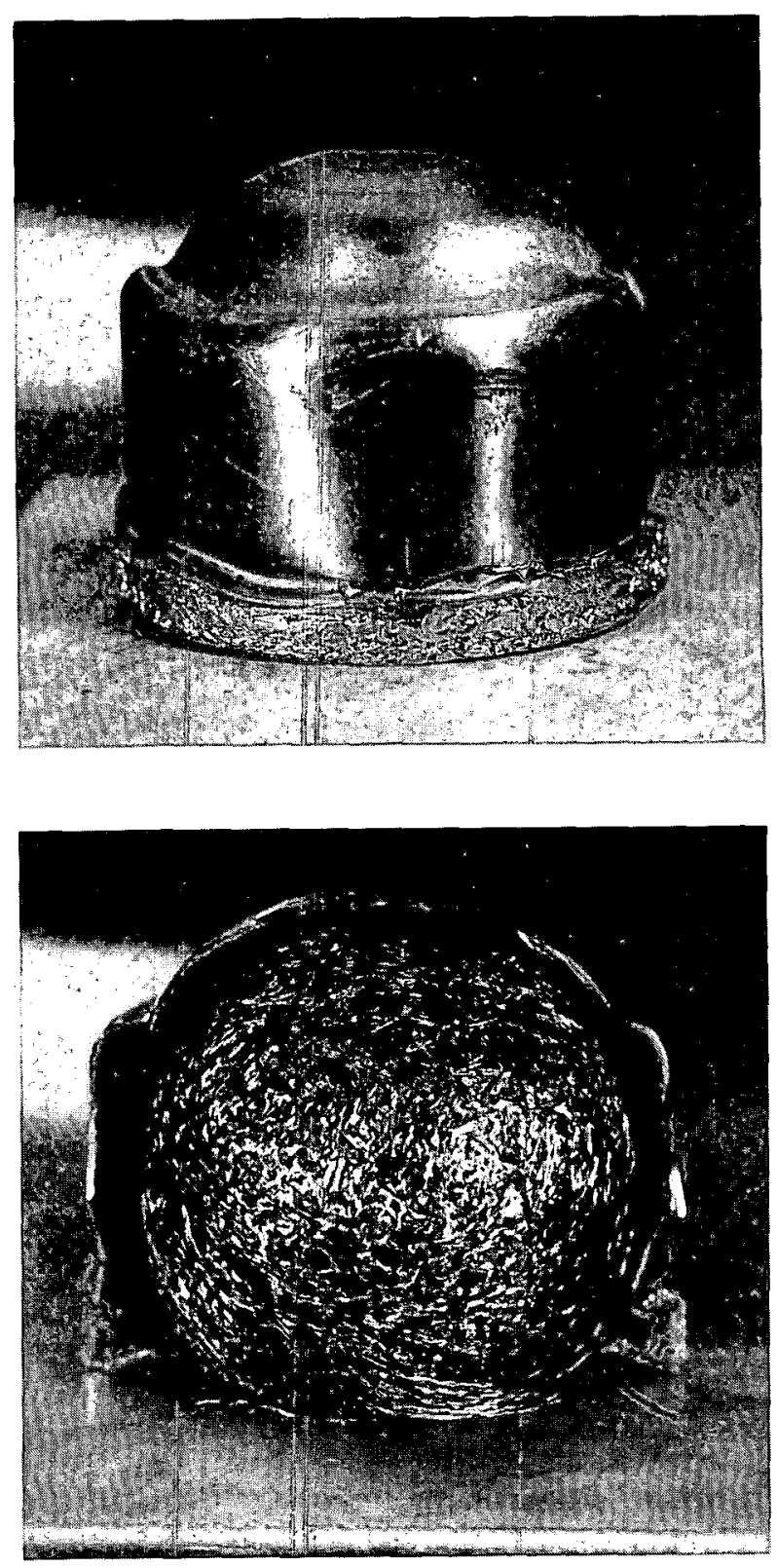

Fig. 25.

MHW sphere in tantalum impact capsule before and after half of the capsule is removed from the heat source.

$\mathrm{ThO}_{2}-\mathrm{Mo}$ cermet simulant fuel, and they were impacted in the large target impact chamber against either a flat cold-rolled C1019 steel target or a structural steel shape. Photography was used to check the heat source orientation at impact. The impact velocities were $46 \mathrm{~m} / \mathrm{s}$, and the impact temperatures were $1100^{\circ} \mathrm{C}$. Figure 26 shows a Viking heat source after impact against an angle iron. 


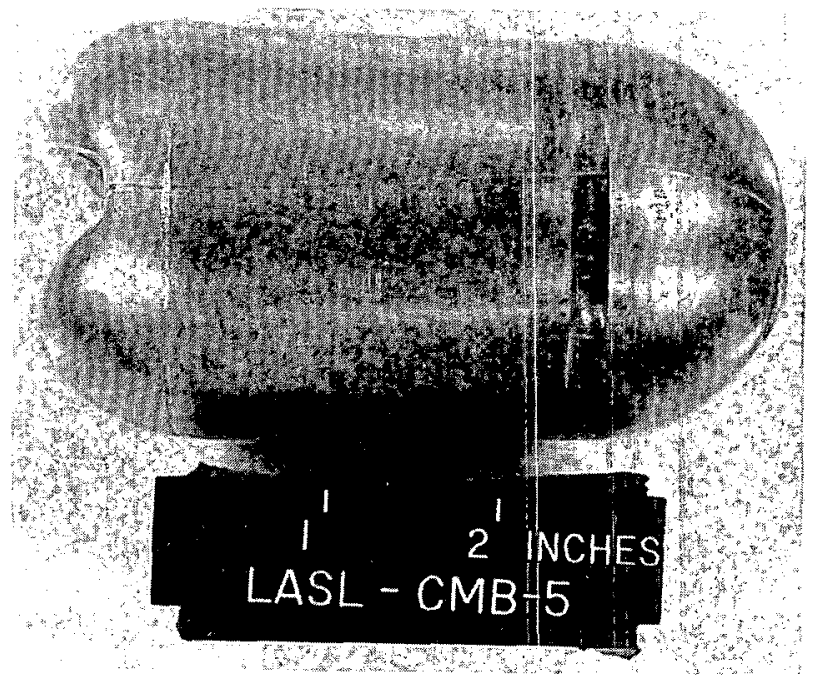

Fig. 26.

Viking heat source after impact against an angle iron.

The projectiles were basically the type incorporating the EB furnace. Governed by the length of the heat source, a four-filament furnace was used instead of the standard three-filament furnace. The furnaces were operated as radiant furnaces instead of $\mathrm{EB}$ furnaces because the impact temperatures were $<1200^{\circ} \mathrm{C}$. With low impact velocities, the OPCs were made of either brass or composite construction.

\section{Plutonia Aerodynamic Disk (PAD)}

PAD is a concept for a radioisotopic heat source that would have a low impact velocity because of its shape. Impact studies were done on a PAD design having a graphite-carbon composite circular disk containing many individually encapsulated $15-\mathrm{Wt}$ ${ }^{238} \mathrm{PuO}_{2}$ heat sources. ${ }^{5}$ One of the functions of the graphite-carbon disk is to protect the individual heat sources during impact.

Many tests were done at room temperature on single, individually encapsulated heat sources containing $\mathrm{UO}_{2}$ simulant fuel. Both bare sources and heat sources protected by graphite or graphitecarbon composite shells were impacted at $25-73 \mathrm{~m} / \mathrm{s}$ velocities against fully hardened $\mathrm{H}-13$ steel. The shapes of the individually encapsulated heat sources were cylindrical, spherical, or hemispherical. Depending upon the impact velocity, either brass or aluminum OPCs were used in the projectiles. High- speed photography of the impacts was used, giving interesting information on the breakup of the graphite and graphite-carbon composite shells and on the release of material through a rupture in the heat-source encapsulation. Figure 27 shows an example of uranium fines being released from an unprotected, ruptured heat source impacted at 58.3 $\mathrm{m} / \mathrm{s}$.

One cylindrical ${ }^{238} \mathrm{PuO}_{2}$ heat source inside a graphite-carbon composite shell was impacted at 38 $\mathrm{m} / \mathrm{s}$ and $90^{\circ} \mathrm{C}$. A special resistance furnace was built to heat the heat source.

Graphite-carbon disks containing several PAD heat sources cannot be impacted in existing impact chambers because of size limitations. Therefore a new impact chamber was recently designed, which should be able to test ${ }^{238} \mathrm{Pu}$ samples with a maximum dimension of $\sim 130 \mathrm{~mm}$. This impact chamber is designed to use flat, fully hardened $\mathrm{H}-13$ or 4340 steel targets, but a shaped steel target could be used in some situations.

\section{Sandia Laboratory Isotope Heat Source}

Several ${ }^{238} \mathrm{PuO}_{2}$ heat sources have been impacted for Sandia Laboratory. These sources have an output of $<10 \mathrm{Wt}$. The typical heat source is a $23-\mathrm{mm}$ diam by $25-\mathrm{mm}-\mathrm{long}$ cylinder with one hemispherical end.

Impacts were done with these heat sources to simulate certain airplane accidents. Impact velocities were usually $150 \mathrm{~m} / \mathrm{s}$, and impact temperatures were $410-450^{\circ} \mathrm{C}$. The standard impact chamber was used with a hardened $\mathrm{H}-13$ steel target. A "Hevi-Duty" type commercial resistance-heating element was used for the projectile furnace. The leading end of the IPC was completely inside the projectile furnace and was machined to hold the heat source at a predetermined orientation. An impact capsule was not used during these tests because of the extremely strong integral encapsulation of the heát source itself.

\section{E. Mechanical Tests}

Various impact tests can be done to study the mechanical behavior of materials and to obtain information for designing isotope heat sources. Impact

*This test was done under severe conditions. The source was not protected by an impact shell, and the impact velocity was higher than typical reentry terminal velocities of 25 to $37 \mathrm{~m} / \mathrm{s}$. 

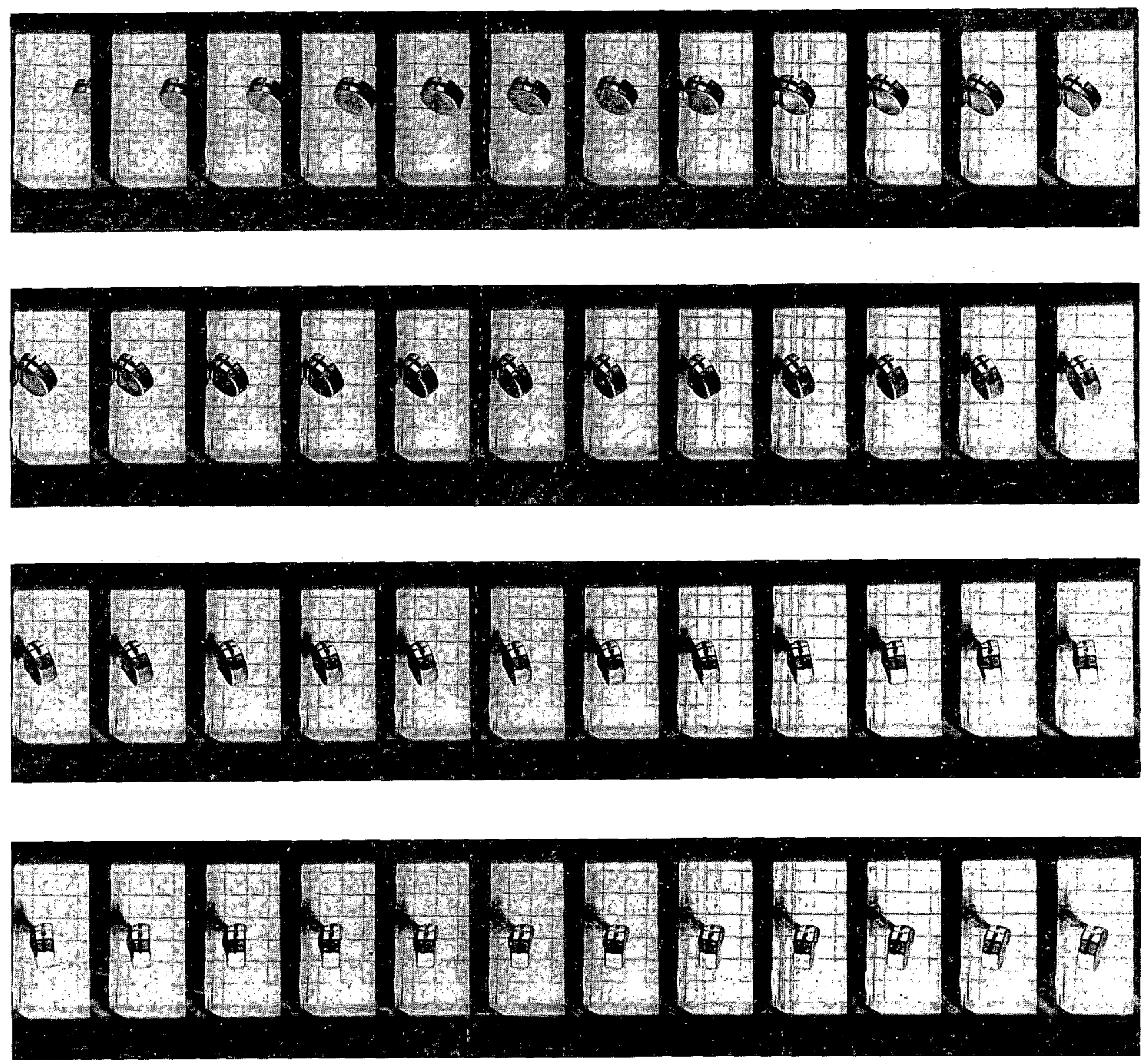

Fig. 27.

High-speed photographs ( $\sim 14000$ half-frames/s) of a bare PAD heat source impacting at 58.3 $\mathrm{m} / \mathrm{s}$ against tool steel. Top strip shows sample striking the target near left border of frames. Following strips show rebound of sample and dispersal of contents. Film is moving from left to right in each strip. 
of an isotope heat source is a mechanical test of a complex system which often gives only limited insight into the impact phenomena because of the complexities. Also, impacting such sources is expensive. Therefore, other approaches are being tried for obtaining information and insight into details of heat source impacts.

1. Engineering Tests. A good structural analysis capability to calculate the impact response of a heat source should lead to improved impact response of future isotope heat sources. For a good structural analysis, the physical phenomena occurring during an impact must be understood and used in computer calculations, and appropriate mechanical properties of the materials in the impacting system must be known. Under impact conditions, heat sources are complex interacting systems with unknown or poorly defined mechanical properties and with properties varying over a large range. Therefore, some simpler, better characterized objects are being impacted to help develop a structural analysis capability.

A set of tests has been proposed and is being done for the MHW system to help develop the structural analysis capability. The tests include impacting 40.6-mm-diam fully annealed copper spheres (bare and with various claddings) against fully hardened $\mathrm{H}-13$ steel. The surface of the $\mathrm{H}-13$ steel is varied to change the frictional characteristics between the bare copper sphere and the target, For clad spheres, gaps between the cladding and the sphere and frictional characteristics between the sphere, cladding, and target are varied. Also, copper spheres inside graphite-carbon composite impact shells are impacted against fully hardened $\mathrm{H}-13$ steel. Whenever possible, well-characterized and well-behaved materials are being used in these tests. Localized strains are measured on many of the surfaces.

2. Fuel Fines Generation. When ${ }^{238} \mathrm{PuO}_{2}$ is impacted, it breaks up into smaller pieces... The amount that the ${ }^{238} \mathrm{PuO}_{2}$, breaks up and the size distribution of the particles have safety implications and are dependent upon the microstructure of the fuel, the impact velocity, the impact temperature, the impact target material, etc. To compare the effects of microstructure, temperature, and velocity, many experimental programs were conducted elsewhere, impacting cylindrical pellets of isotope fuel against flat surfaces; after each test, the size distribution of the fuel particles is determined.

In a typical fines-generation test, ${ }^{6}$ the fuel pellet is in a capsule which is accelerated upward in a gas gun into an impact chamber. The fuel rests on the bot- tom of the capsule until the capsule hits a stopping fixture inside the impact chamber. The stopping fixture stops the capsule through a distance of $\sim 10-30$ $\mathrm{mm}$ of capsule travel. While the capsule is being stopped, the fuel pellet inside the capsule goes into free flight at the maximum capsule velocity, striking the top of the capsule after the capsule is stopped. The fuel is then recovered from the capsule to determine particle size distribution.

This fines-generation test mode has been adapted to IFIT. The capsule (small capsule, Fig. 10) is made of tantalum-10\% tungsten alloy. Except for a few minor modifications, the projectile is the same as that used for MHW impacts. The standard impact chamber is used with the stopping fixture inside the inner catch tube. The equipment has been satisfactorily tested at $100 \mathrm{~m} / \mathrm{s}$ and $1370^{\circ} \mathrm{C}$.

There are several objections to the way in which the fines test is done. A critical balance exists between the length of the capsule and the manner of stopping the capsule because the pellet may strike the top of the capsule before the capsule is completely stopped. Also, the top of the capsule is the impact surface and is not a rigid surface at higher temperatures. Finally, the capsule may tilt as it is stopped by the stopping fixture, and the fuel pellet would strike a skewed surface. We are developing a system that will minimize or eliminate these problems. The fuel pellet will be in a capsule with $0.5-\mathrm{mm}$-thick end walls so that both ends of the capsule will touch the fuel pellet. The capsule will have a void volume radially about the fuel pellet; radial expansion of the fuel pellet will be accommodated by the void volume at impact. The impact will be against a rigidly supported piece of tool steel.

\section{SUMMARY}

The LASL IFIT has proven to be a versatile facility. Several types of heat sources containing ${ }^{238} \mathrm{PuO}_{2}$ have been impacted safely under diverse conditions. Impact velocities have ranged from 25 to $150 \mathrm{~m} / \mathrm{s}^{*}$ and impact temperatures have been from room temperature to $>1400^{\circ} \mathrm{C}$. The object size that can be impacted is limited to $\sim 130 \mathrm{~mm}$ in the radial plane of the gun; obviously, larger objects can be impacted if the larger dimensions are alined along the length of the gun. Normally, no fasteners are required to hold the specimen to the projectile because the gun fires vertically upward, and gravity is used to hold the specimen on the projectile.

*Although the gas gun was designed to achieve velocities of $300 \mathrm{~m} / \mathrm{s}$ under proper conditions, no need has arisen yet for a $300-\mathrm{m} / \mathrm{s}$ muzzle velocity. 
The success of the modular structure has been particularly noteworthy with respect to the impact chamber. When impacting ${ }^{238} \mathrm{PuO}_{2}$ heat sources with maximum dimensions of $\sim 65.75 \mathrm{~mm}$, the standard impact chamber can be used with an inner catch tube as the primary containment. Targets that can be used in the inner catch tube include granite, steel, concrete, and special stopping fixtures. Because of interest in impacts against large structural members and soil (which must be present in a sizable volume to retain its properties), the large target impact chamber was developed for testing materials with low hazard levels, such as thoria and natural urania. A new impact chamber is being designed and built to accommodate larger ${ }^{238} \mathrm{PuO}_{2}$ heat sources.

High-speed photography is available for im. mediate use with nonplutonium impacts. Flash xray photography is also available but has not been used.

One of the most valuable uses of IFIT is impacting well-characterized models, to develop and verify computer calculations of impact response. The ultimate success of this effort will permit calculations of the impact behavior of proposed heat-source designs, thus reducing expensive design verification testing.

The major limitation of IFIT is the time required to conduct the tests. When the samples contain plutonium, only $2-3$ tests can be run in a 40 -h week. This limitation is primarily caused by the need for multiple containment, each stage of which requires additional time. We feel, however, that the ability to test radioactive fuels instead of simulants far outweighs the time limitations.

\section{REFERENCES}

1. William R. Corliss and Robert L. Mead, "Power from Radioisotopes," An Understanding the Atom Series Booklet, U.S. Energy Research and Development Administration, Division of Technical Information Extension, Oak Ridge, TN (1971).

2. Larry O. Seamons, "Isotope Fuel Impact Facility," Sandia Laboratories, Albuquerque, report SC-DC-70-4991 (1970).

3. "Multi-Hundred Watt Radioisotope Thermoelectric Generator Program, Final Safety Analysis Report, Vol. I," General Electric document GEMS419 (March 1975).

4. "SNAP 19 Viking, Final Safety Analysis Report. Vol. I," Teledyne Isotopes document ESD-3069-15-1 (August 1974).

5. James J. Burns and J. W. Vorreiter, "Plutonia Aerodynamic Disk (PAD) Summary Report," Los Alamos Scientific Laboratory report LA-5527-MS (May 1974).

6. W. J. Dalby, "Status Report. Impact Tests: Radioisotope Fuels and Simulants," Sandia Laboratories, Albuquerque, report SC-DR-710 218 (April 1971).

\section{APPENDIX A}

\section{CALCULATING INITIAL BREECH PRESSURE}

A relationship is derived that predicts the projectile velocity, u.ing the mass of the projectile and the initial pressure of the propulsion gas in the breech. The relationship is rearranged for calculating the breech pressure from the projectile mass and the desired projectile velocity. Several assumptions are made in the derivation. The net effect of the assumptions is measured by how well the relationship predicts the projectile velocity. In this comparison process we have found that if one of the constants in the relationship is slightly changed, the relationship is adequate except for high velocity projectiles.

The person who originally derived the relationship is an unknown author from Lockheed, and consequently proper recognition cannot be given. Because this useful relationship has not been documented in the open literature, our intent is to make it available.

\section{NOMENCLATURE}

$$
\begin{aligned}
& A_{1}=\underset{\text { breech }}{1 / 4 \pi D_{1}^{2}}=\text { cross-sectional area oi } \\
& A_{2}=1 / 4 \pi D_{2}^{2}=\text { cross-sectional area of } \\
& \text { bore } \\
& D_{1}=\text { breech diameter } \\
& \mathrm{D}_{2}=\text { bore diameter } \\
& \mathbf{E}=\text { internal energy of the propulsion gas }
\end{aligned}
$$


$\mathrm{KE}=$ combined kinetic energy of the propulsion gas and the projectile

$L_{1}=$ breech length

$L_{2}=$ bore lerigth

$m_{p}=$ projectile mass

$M=$ molecular weight of the propulsion gas

$P_{0}=$ initial breech pressure

$R$ = ideal gas constant

$t=\operatorname{time}$

$T_{0}=$ initial absolute temperature of the propulsion gas

$u=v e l o c i t y$ of the propulsion gas

$u_{1}=$ velocity of the propulsion gas in the breech at the bore-breech transition

$u_{2}=$ velocity of the propuision gas in the bore at the bore-breech transition

$v_{0}=$ initial specific volume (rnass in moles) of the propulsion gas

$V_{1}=L_{1} A_{1}=$ volume of the isreech

$y=$ ratio of the heat capacity of the propulsion gas at constant pressure to that at constant volume

$\eta=\left\{1+\frac{A_{2} \xi_{p}}{V_{1}}\right\}^{-1}$

$\xi_{1}=$ coordinate for the breech (Fig. A-1)

${ }_{2}=$ coordinate for the bore (Fig. A-I)

$\xi_{p}=$ coordinate for the projectile in the bore (Fig. A-1)

$\rho_{0}=$ initial density of the propulsion gas in the breech

$p=$ density of the propulsion gas.

\section{ASSUMPTIONS}

The following are assumed in the derivation.

1. The density of the propulsion gas is a function of time but not of position.

2. A discontinuity in the propulsion gas velocity exists at the breech-bore transition as follows:

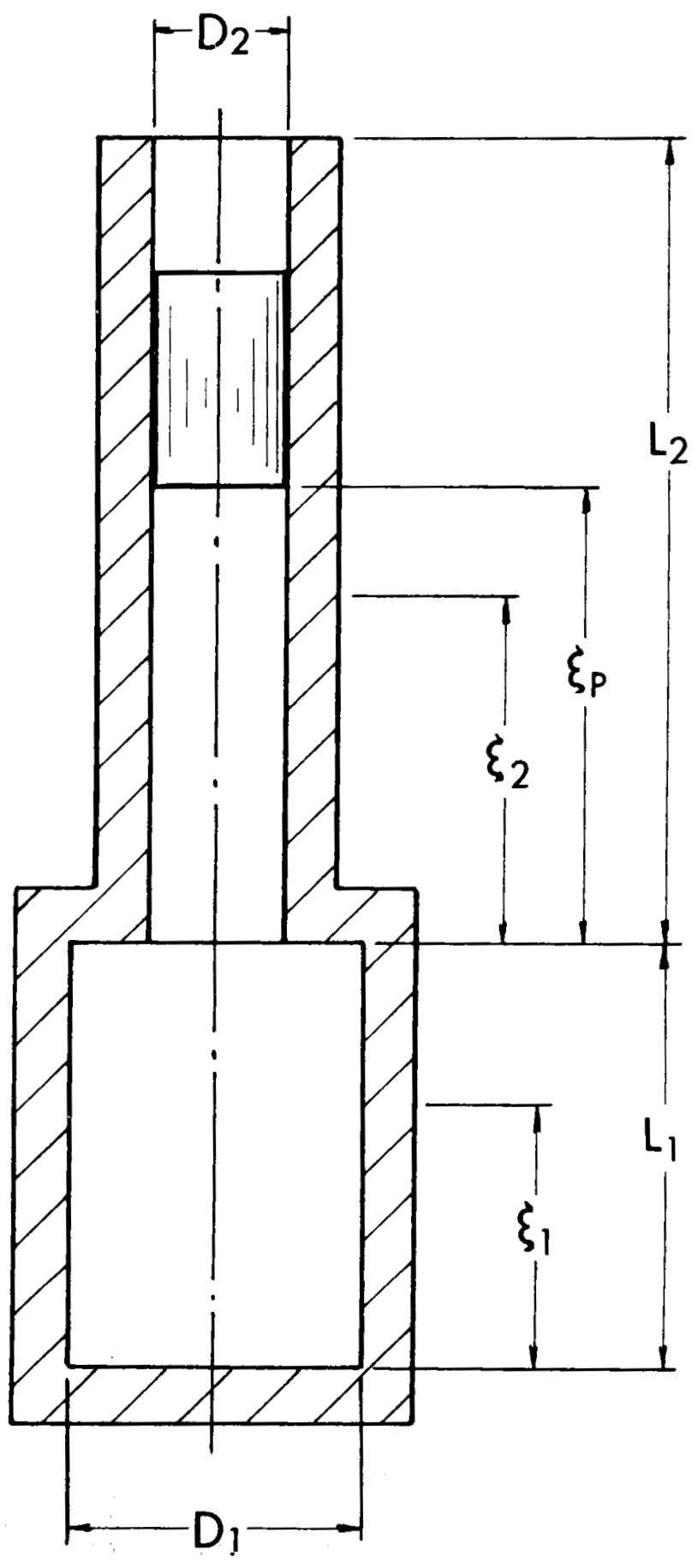

Fig. A-1.

Gun variables for mathematical derivation. 


$$
\mathrm{u}_{1}=\frac{\mathrm{A}_{2}}{\mathrm{~A}_{1}} \cdot \mathrm{u}_{2} .
$$

3. The propulsion gas is an ideal gas.

4. The propulsion gas expands isentropically.

5. No friction exists between the barrel and the projectile.

6. The change in the internal energy, $\Delta E_{1}$, of the propulsion gas from its initial internal energy is equal to the combined kinetic energy of the propulsion gas and the projectile.

7. The fluid flow is time-dependent and onedimensional.

8. There is no gas pressure in front of the projectile.

\section{DERIVATION}

Several relationships will be derived and taken from thermodynamics. These relationships will be useful in the energy balance implied by assumption 6.

The equation in an Eulerian reference frame for the conservation of mass for one-dimensional fluid flow, with $\rho$ independent of $\xi$, is

$$
\frac{d \rho}{d t}+\rho \frac{\partial u}{\partial \xi}=0,
$$

or

$$
\frac{\partial u}{\partial \xi}=f(t)
$$

and

$$
\left.\frac{\partial u}{\partial \xi_{1}}\right|_{\xi_{1}}=L_{1}=\left.\frac{\partial u}{\partial \xi_{2}}\right|_{\xi_{2}}=0
$$

for a given time because $\rho$ is not a function of position. Integrating Eq. (A-2) with the boundary condition

$$
\left.u\right|_{1=0}=0
$$

and inserting the values of $\mathrm{u}_{1}, \mathrm{u}_{2}$, and $\mathrm{u}_{\mathrm{p}}$ gives

$$
u\left(\xi_{1}\right)=\frac{u_{1}}{L_{1}} \xi_{1} ;
$$

and

$$
u\left(\xi_{2}\right)=u_{2}+\frac{\left(u_{p}-u_{2}\right)}{\xi_{p}}=\xi_{2} .
$$

Differentiating Eqs. (A-4) and (A-5) with respect to $\xi_{1}$ and $\xi_{z}$, respectively, and substituting into $\mathrm{Eq}$. (A3) gives

$$
\frac{u_{1}}{L_{1}}=\frac{u_{p}-u_{2}}{\xi_{p}} .
$$

Solving for $\mathrm{u}_{1}$ and $\mathrm{u}_{2}$ using Eq. (A-6) and assumption 2 yields

$$
u_{1}=\frac{\left(A_{2} / A_{1}\right) u_{p}}{1+\frac{A_{2}{ }^{s} p}{V_{1}}}=\left(\frac{A_{2}}{A_{1}}\right) T_{i} u_{p}
$$

and

$$
u_{2}=\frac{u_{p}}{1+\frac{A_{2} p_{p}}{V_{1}}}=\eta_{p}
$$

Using assumption 1 and the conservation of mass for the propulsion gas,

$$
\rho_{0} V_{1}=\left(V_{1}+A_{2} F_{p}\right) \rho
$$

which can be rearranged as

$$
p=\frac{\rho_{0}}{1+\frac{A_{2} \bar{s}_{p}}{V_{1}}}=n \rho_{0} \text {. }
$$

Finally, from thermodynamics the following relationship holds for the change in internal energy $\triangle \mathrm{E}$ for an isentropic expansion (assumption 4) of an ideal gas (assumption 3):

$$
\Delta E=\frac{D_{0} V_{1}}{\gamma-1} 1-\left[\frac{1}{\left(1+A_{2} s_{p} / V_{1}\right)^{\gamma-1}}\right] \text {. }
$$

where the change in internal energy is relative to the initial internal energy of the propulsion gas. The key relations which are used are Eqs. (A-4)-(A-10).

The combined kinetic energy of the projectile and the propulsion gas is

$$
K E=\frac{m_{p} u^{2}}{2}+\frac{\rho A_{1}}{2} \int_{0}^{L_{1}} \frac{u_{1}^{2}}{L_{1}^{2}} \xi_{1}^{2} d \xi_{1}
$$




$$
+\frac{\rho A_{2}}{2} \int_{0}^{\xi_{p}}\left[u_{2}+\left(\frac{u_{p}-u_{2}}{\xi_{p}}\right) \xi_{2}\right]^{2} d \bar{s}_{2},
$$

which upon integration becomes

$$
K E=\frac{m_{p_{p} u^{2}}}{2}+\frac{\rho V_{1} u_{1}^{2}}{6}+\frac{\rho A_{2}{ }^{5} p\left(u_{p}^{3}-u_{2}^{3}\right)}{6\left(u_{p}-u_{2}\right)} .
$$

Noting that

$$
\frac{u_{p}^{3}-u_{2}^{3}}{u_{p}-u_{2}}=u_{p}^{2}-u_{p} u_{2}+u_{2}^{2} \text {. }
$$

and substituting Eqs. (A-7) -- (A-9) into the kinetic energy equation gives

$$
\begin{aligned}
K E & =u_{p}{ }_{p}\left\{\frac{m_{p}}{2}+\frac{\rho_{0} V_{1} \eta^{3}}{6}\left(\frac{A_{2}}{A_{1}}\right)^{2}\right. \\
& \left.+\frac{\rho_{0} A_{2} \gamma^{\eta}}{6}\left(1+\eta+\eta^{3}\right)\right\}=u_{p} 2\left\{\frac{m_{p}}{2}\right. \\
& \left.+\frac{\rho_{0} V_{1} \eta^{3}}{6}\left[\left(\frac{A_{2}}{A_{1}}\right)^{2}+\frac{A_{2}{ }^{5}}{V_{1}}\left(\frac{1}{\eta^{2}}+\frac{1}{\eta}+1\right)\right]\right\} .
\end{aligned}
$$

Substituting for $\eta$ and collecting terms,

$$
\begin{aligned}
\mathrm{KE} & =u_{\mathrm{p}}^{2}\left\{\frac{\mathrm{m}_{\mathrm{p}}^{2}}{2}+\frac{1 / 6 \mathrm{p}_{0} \mathrm{~V}_{1}}{\left(1+\frac{\mathrm{A}_{2}{ }_{\mathrm{p}}}{\mathrm{V}_{1}}\right)^{3}}[-1+1\right. \\
& +3\left(\frac{\mathrm{A}_{2}{ }^{\xi} \mathrm{p}}{\mathrm{V}_{1}}\right)+3\left(\frac{\mathrm{A}_{2} \xi_{\mathrm{p}}}{\mathrm{V}_{1}}\right)^{2}+\left(\frac{\mathrm{A}_{2} \xi_{p}}{\mathrm{~V}_{1}}\right)^{3} \\
& \left.\left.+\left(\frac{\mathrm{A}_{2}}{\mathrm{~A}_{1}}\right)^{2}\right]\right\} .
\end{aligned}
$$

and noting that this expression can be simplified by the algebraic identity of the form

$$
(1+a)^{3}=1+3 a+3 a^{2}+a^{3}
$$

we obtain

$$
K E=\frac{u_{p}^{2}}{2}\left\{n_{p}+\frac{\rho_{0} V_{1}}{3}\left[1-\frac{1-\left(\frac{A_{2}}{A_{1}}\right)^{2}}{\left(1+\frac{A_{2} p_{p}}{V_{1}}\right)^{3}}\right]\right\}
$$

By assumption 6, Eqs. (A-10) and (A-11) can be equated, and after solving for $u_{p}{ }^{2}$, we obtain

$$
u_{p}^{2}=\frac{\frac{2 \rho_{0} V_{1}}{m_{p}^{(\gamma-1)}}\left[1-\frac{1}{\left(1+A_{2} \bar{s}_{p} / V_{1}\right)^{\gamma-1}}\right]}{1+\frac{\rho_{0} V_{1}}{3 m_{p}}\left[1-\frac{1-\left(A_{2} / A_{1}\right)^{2}}{\left(1+A_{2} \xi_{p} / V_{1}\right)^{3}}\right]} .
$$

$(A-12)$

The author at Lockheed noted that if the factor of $1 / 3$ in the denominator term is replaced by $0.413, \mathrm{Eq}$. (A-12) agrees satisfactorily with experimental data for projectile velocities up to 1.5 times the initial sound speed of the propulsion gas, air. Our experience with various gas guns and propulsion gases essentially agrees with these results, providing the initial breech pressure is increased by a small increment to compensate for projectile-bore friction. For projectiles with $\mathrm{O}$-ring seals, we have found that increasing the breech pressure $70-140 \mathrm{kPa}$ is normally adequate compensation for friction.

Usually, the mass of the projectile and the desired projectile velocity are known, and the breech pressure is unknown. After $\rho_{0}$ is expressed in terms of $P_{0}, E q .(A-12)$ can be solved for $P_{0}$, using the ideal gas law: The ideal gas law, $\mathrm{Pv}=\mathrm{RT}$, can be rearranged to

$$
\rho_{0}=\frac{M}{v_{0}}=\frac{M p_{0}}{R T_{0}}
$$

Substituting $\rho_{0}$ into Eq. (A-12), using 0.413 instead of $1 / 3$, and solving for $P_{o}$ gives 


$$
\begin{aligned}
P_{0} & =\frac{m_{p}{ }^{2} p}{V_{1}}\left\{\frac{2}{\gamma-1}\left[1-\frac{1}{\left(1+A_{2} \xi_{p} / V_{1}\right)^{\gamma-1}}\right]\right. \\
& -0.413 u_{p}^{2} \frac{M}{R T_{0}}\left[1-\frac{1-\left(A_{2} / A_{1}\right)^{2}}{\left(1+A_{2} \xi_{p} / V_{1}\right.}\right]^{-1}
\end{aligned}
$$

where, as just mentioned, a small increment of pressure must be added to this calculated pressure to compensate for projectile-bore friction.

\section{APPENDIX B}

\section{VELOCITY PREDICTION AND CONTROL}

Equations (A-12) and (A-13), App. A, are useful for predicting projectile velocities in various gas guns. These relationships were used to design the gas gun for IFIT and have been used successfully in operating the facility. However, some precautions must be taken before the relationships become useful. As stated in App. A, the relationships assume there is no friction between the gun bore and the projectile, and the gun bore is evacuated in front of the projectile. In IFIT the gun bore is evacuated in front of the projectile, but keeping the barrelprojectile friction small is troublesome. Three ways to reduce barrel-projectile friction are used.

- The 0-ring séal for the projectile usually has 0.30$0.36 \mathrm{~mm}$ of compression for a $6.985-\mathrm{mm}$ wall $\mathrm{O}$-ring. This compression, which is much smaller than normally used in O-ring seals, has worked very well.

- Second, the type of material used in the O ring affects the barrel-projectile friction. Lack of detailed information precludes formulation of firm guidelines for choosing the proper $\mathrm{O}$-ring material under all circumstances; for the IFIT gas gun, high-durometer buta- $\mathrm{N}$ O-rings were satisfactory in reducing projectile-barrel friction.

- The third way of reducing barrel-projectile friction is to lubricate the gun bore. In addition to having good lubricating properties, the lubricant must have a low vapor pressure so that it does not degrade the vacuum and also so that it remains on the gun bore. Greases have been found to be better barrel lubricants than oils because they are more effective lubricants in thin layers and at high bearing pressures and have lower vapor pressures. Lubriplate $630-\mathrm{AA}$ is normally used to grease the IFIT barrel.

Another factor in controlling the projectile velocity is controlling the ratio of kinetic energy of the projectile to the energy dissipated by barrelprojectile friction. As explained in Sec.II.B.1, the ratio should be large. At low projectile velocities, the ratio is made large by using an OPC with a large mass. At high projectile velocities, a low-mass OPC is used because a large ratio is obtained primarily by the high projectile velocity. 